TRANSACTIONS OF THE

AMERICAN MATHEMATICAL SOCIETY

Volume 348, Number 7, July 1996

\title{
ON COMPLETE NONORIENTABLE MINIMAL SURFACES WITH LOW TOTAL CURVATURE
}

\author{
FRANCISCO J. LOPEZ
}

\begin{abstract}
We classify complete nonorientable minimal surfaces in $\mathbb{R}^{3}$ with total curvature $-8 \pi$.
\end{abstract}

\section{INTRODUCTION}

Complete minimal surfaces of finite total curvature in three dimensional Euclidean space are interesting objects from the point of view of classical Differential Geometry.

Some basic structure properties of these surfaces where described first by $\mathrm{R}$. Osserman [OS], and later, by Jorge and Meeks [JM].

Until recently few classification results have been obtained.

Fixing the value of the total curvature, we know in the orientable case:

- The Catenoid and Enneper surfaces are the unique complete minimal surfaces with total curvature $-4 \pi$, by R. Osserman [OS].

- The only complete orientable minimal surface of total curvature $-8 \pi$ and non trivial topology is the genus one surface exhibited by Chen and Gackstatter (see $[\mathrm{CG}]$ ). This result was obtained independently by Bloss [BL] and López [L1]. See also Barbanel's work [B] or [L1] for the study of complete genus zero orientable minimal surfaces of total curvature $-8 \pi$.

In the nonorientable case, these results are known:

- The only complete nonorientable minimal surface with total curvature $-6 \pi$ is Meeks' Möbius strip (see [M]).

- The classification of complete minimal once punctured projective planes with total curvature $-10 \pi$, by T. Ishihara [I1].

- The classification of complete minimal twice punctured projective planes with total curvature $-10 \pi$, by R. Kusner [K2]. See also [S].

Other examples of nonorientable minimal surfaces can be found in Kusner [K1], Barros [BA], Ishihara [I2], Oliveira [OL], Oliveira and Toubiana [OT] and [SM].

The author exhibited a complete minimal once punctured Klein Bottle with total curvature $-8 \pi$ (see [L2]). In this paper we present a uniqueness theorem for this surface, classifying it as the only complete nonorientable minimal surface in $\mathbb{R}^{3}$ with total curvature $-8 \pi$ (see Section 3 ).

Received by the editors March 20, 1995.

1991 Mathematics Subject Classification. Primary 53A10; Secondary 53C42.

Research partially supported by DGCYT grant No. PB91-0731.

(C)1996 American Mathematical Society 
We also study the symmetry group of complete nonorientable minimal surfaces with critical total curvature (from the point of view of the Jorge-Meeks formula) and double cover of hyperelliptic type and even genus. We obtain that this group contains at most four symmetries. Moreover, we prove that the Klein Bottle in [L2] is the only complete nonorientable minimal surface with non trivial topology of total curvature greater than $-12 \pi$ and at least three symmetries. For more details, see Section 3.

The fundamental tools used, apart from Osserman's classic theorems, are: A congruence formula due to Meeks for the topology and the total curvature of nonorientable minimal surfaces (see $[\mathrm{M}]$ ), together with the Jorge-Meeks formula (see $[\mathrm{JM}]$ ) and the Weierstrass representation for nonorientable minimal surfaces (see $[\mathrm{M}])$.

We also use a nonexistence theorem for complete nonorientable minimal surfaces with two embedded ends, by R. Kusner [K1].

The uniqueness theorems contained in this paper, together with the results in [L1] or [BL], yield the classification of complete minimal surfaces in $\mathbb{R}^{3}$ with total curvature $-8 \pi$.

This is the final version of a preprint with the same name. The author has done a considerable effort in order to get simplicity and clarity. I wish to thank Francisco Martin for useful conversations about these results.

It could be interesting to notice that recently F. Martin and the author (see [LM]) have constructed a new family of complete nonorientable minimal surfaces with arbitrary genus $k \geq 2$ (that is, with Euler characteristic of the associated closed surface $2-k$ ), one end and large symmetry group. This construction leads to the minimal once punctured Klein Bottle in [L2] for $k=2$.

\section{Preliminaries on minimal Surfaces}

In this section we recall some basic results about complete minimal surfaces of finite total curvature.

Let $x_{0}: M \rightarrow \mathbb{R}^{3}$ be a minimal immersion in three dimensional Euclidean space $\mathbb{R}^{3}$ of an orientable surface $M$.

$M$ has in a natural way the structure of a Riemann surface, and we denote by $g$ and $\eta$ the meromorphic function and the holomorphic 1-form determined by the Weierstrass representation of $x_{0}$ (see [OS]).

It is well known that, modulo natural identifications, $g$ is the Gauss map of $M$. Moreover,

$$
x_{0}=\operatorname{Real} \int\left(\phi_{1}, \phi_{2}, \phi_{3}\right)
$$

where $\phi_{1}=\frac{\eta}{2}\left(1-g^{2}\right), \phi_{2}=i \frac{\eta}{2}\left(1+g^{2}\right), \phi_{3}=n g$ are holomorphic 1-forms on $M$ satisfying $\sum_{k=1}^{3}\left|\phi_{k}\right|^{2} \neq 0$.

In particular, the 1 -forms $\phi_{k}, k=1,2,3$, have no real periods on $M$.

Assume now that $x: N \rightarrow \mathbb{R}^{3}$ is a nonorientable minimal surface in $\mathbb{R}^{3}$. We can define a Weierstrass type representation for $N$ as follows:

Let $\pi_{0}: M \rightarrow N$ be the conformal oriented two-sheeted cover of $N$, and take $I: M \rightarrow M$ the antiholomorphic order two deck transformation for this cover. Put $(g, \eta)$ the Weierstrass data of $x_{0}=x \cdot \pi_{0}$.

Consider now the antiholomorphic involution $\mathcal{I}$ in the Riemann sphere $\overline{\mathbb{C}}=$ $\mathbb{C} \cup\{\infty\}$ defined by $\mathcal{I}(z)=-1 / \bar{z}, z \in \overline{\mathbb{C}}$, and write as usual $\pi: \overline{\mathbb{C}} \rightarrow \mathbb{R P}^{2}=\overline{\mathbb{C}} /\langle\mathcal{I}\rangle$, 
the natural two sheeted cover the the Projective Plane, where $\langle\mathcal{I}\rangle=\{1, \mathcal{I}\}(1$ represents the identity map in $\overline{\mathbb{C}}$ ).

As $x_{0} \cdot I=x_{0}$, from (1) we deduce that $I^{*}\left(\phi_{k}\right)=\bar{\phi}_{k}, k=1,2,3$, that is

$$
g \cdot I=\mathcal{I} \cdot g \text { and } \overline{I^{*}(\eta)}=-\eta g^{2} .
$$

Conversely, given the Weierstrass representation $(M, g, \eta)$ of an orientable minimal immersion $x_{0}$, and given I an antiholomorphic involution without fixed points on $M$ satisfying (1.2), then $x_{0}$ induces a minimal immersion $x$ of $N=M /\langle I\rangle$ in $\mathbb{R}^{3}$ such that $x_{0}=x \cdot \pi_{0}$.

We call $(M, I, g, \eta)$ the Weierstrass representation of $x$. For more details, see Meeks $[\mathrm{M}]$.

Observe that from (1.2), we can define the "Gauss map" of the nonorientable immersion $x$ as the unique conformal map $G: N \rightarrow \mathbb{R} \mathbb{P}^{2}$ satisfying $G \cdot \pi_{0}=\pi \cdot g$.

For the remainder, we suppose $x: N \rightarrow \mathbb{R}^{3}$ is complete and has finite total curvature $C(N)$.

Then, Huber proved that $M$ is conformally diffeomorphic to a compact Riemann surface $\bar{M}$ punctured in a finite number of points $\left\{P_{1}, \ldots, P_{r}, I\left(P_{1}\right), \ldots, I\left(P_{r}\right)\right\}$, and Osserman that the Weierstrass data extends meromorphically to $\bar{M}$. In particular, $N$ is conformally diffeomorphic to $\bar{N}-\left\{\bar{P}_{1}, \ldots, \bar{P}_{r}\right\}$, where $\bar{N}=\bar{M} /\langle I\rangle$ and $\bar{P}_{i}=$ $\pi_{0}\left(P_{i}\right), i=1, \ldots, r$.

Therefore, the Gauss map $g$ (or $G$ ) has a well defined degree and the total curvature $C(N)$ is a multiple of $-2 \pi$. In fact, $C(N)=-2 \pi n$, where $n=\operatorname{Degree}(G)=$ $\operatorname{Degree}(g)$.

We have stronger restrictions on the topology of $N$. Meeks $[\mathrm{M}]$ showed that the Euler characteristic $\chi(\bar{N})$ of $\bar{N}$ and $n=-C(N) / 2 \pi$ are congruent modulo 2 .

Jorge and Meeks [JM] showed that the asymptotic behavior of $M$ around each end $P_{i}\left(\right.$ or $\left.I\left(P_{i}\right)\right)$ is determined by an integer number $\nu_{i}$ greater than one and

$$
2 n=-\chi(\bar{M})+2\left(\sum_{i=1}^{r}\left(\nu_{i}+1\right)\right) .
$$

In other words,

$$
n=-\chi(\bar{N})+\sum_{i=1}^{r}\left(\nu_{1}+1\right) .
$$

The numbers $\nu_{i}$ can be computed via the Weierstrass representation as follows:

$$
\nu_{i}=\operatorname{Maximum}\left\{\operatorname{Ord}\left(\phi_{k}, P_{i}\right), k=1,2,3\right\}-1, \quad i=1, \ldots, r,
$$

where as before $n$ is the degree of the Gauss map $G$ of $x$.

Assume that $N$ is not the covering of any minimal surface and write $\operatorname{Iso}(N)$ for the isometry group of $N$. Then, denote by $\operatorname{Sym}(N)$ the subgroup of $\operatorname{Iso}(N)$ consisting of those isometries which are restrictions of rigid motions in $\mathbb{R}^{3}$ leaving $x(N)$ invariant. Calabi proved that $\operatorname{Iso}(N)=\operatorname{Sym}(N)$ if there exists $j \in\{1,2,3\}$ such that $\phi_{j}$ is not exact. A complete discussion of this subject can be found in $[\mathrm{HM}]$.

\section{Some Remarks on compact Riemann surfaces}

In this section we set forth some basic facts about the classical theory of compact Riemann surfaces. We refer for more details to $[\mathrm{FK}]$ or other related books. 
Let $\bar{M}$ be a compact Riemann surface of genus $k \geq 1$. Denote by $\operatorname{Div}(\bar{M})$ the group of divisors of $\bar{M}$. As usual, degree $(D)$ is the degree of $D \in \operatorname{Div}(\bar{M})$. For $D_{1}, D_{2} \in \operatorname{Div}(\bar{M}), D_{1} \geq D_{2}$ when $D_{1} / D_{2}$ is an integral divisor. Given $f$ a meromorphic function on $\bar{M},[f]_{0},[f]_{\infty},[f]$ will denote the zero divisor, the polar divisor and the principal divisor of $f$, respectively. Remember that $[f]=[f]_{0} /[f]_{\infty}$. Analogously, if $\omega$ is a meromorphic 1 -form on $\bar{M}$, we define $[\omega]_{0},[\omega]_{\infty}$ and $[\omega]=$ $[\omega]_{0} /[\omega]_{\infty}$, the zero divisor, polar divisor and canonical divisor of $\omega$, respectively. Given $D_{1}, D_{2} \in \operatorname{Div}(\bar{M})$, we say that $D_{1}$ and $D_{2}$ are equivalent, and write $D_{1} \sim D_{2}$, if and only if $D_{1} / D_{2}$ is principal (that is, $D_{1} / D_{2}=[f]$, where $f$ is a meromorphic function on $\bar{M})$.

Given $\omega_{1}, \omega_{2}$ meromorphic 1 -forms in $\bar{M}$, we say that they are equivalent, and write $\omega_{1} \approx \omega_{2}$, if and only if $\omega_{1}-\omega_{2}$ is exact (that is, $\omega_{1}-\omega_{2}=d f$, where $f$ is a meromorphic function on $\bar{M})$. $\bar{M}$.

In what follows, Aut $(\bar{M})$ denotes the group of holomorphic automorphisms of

Suppose there exists $I: \bar{M} \rightarrow \bar{M}$, an antiholomorphic involution without fixed points on $\bar{M}$. Assume that $k \geq 2$ and $\bar{M}$ is hyperelliptic.

Then label by $A: \bar{M} \rightarrow \bar{M}$ the only holomorphic involution with $2 k+2$ fixed points on $\bar{M}$. The uniqueness of $A$ gives $A=I \circ A \circ I$. For $k$ even, it is not hard to see that $A \circ I$ has infinitely many fixed points on $\bar{M}$. However, for $k$ odd, this does not happen in general (see $[\mathrm{R}]$ ).

In the elliptic case $k=1$, that is, when $\bar{M}$ is a conformal torus $T$, we have a real one-parameter family of holomorphic involutions $A$ on $T$ with four fixed points satisfying $I \circ A \circ I=A$. Since the Klein Bottle $T /\langle I\rangle$ is not conformally homogeneous, given $P \in T$ any point, we can not assure $A(P)=P$ for some holomorphic involution $A$ in the above family. However, we can prove the following proposition:

Proposition 2.1. There exists a unique holomorphic involution $A$ on $T$ with four fixed points satisfying: $A \circ I=I \circ A, A \circ I(P)=P$.

Proof. The Riemann-Roch Theorem yields the existence of a degree two meromorphic function $s$ on $T$ verifying: $s(P)=s(I(P))=\infty$. As $s$ is determined up to adding constants and homotheties, and $I \circ I=\mathrm{Id}$, it is easy to prove that $s \circ I=\lambda \bar{s}+\mu$, where $\lambda, \mu \in \mathbb{C}$ and $|\lambda|=1, \lambda \bar{\mu}+\mu=0$. Even more, up to a suitable change of parameter, we can put $s \circ I=-\bar{s}$. Consider $A$ the only nontrivial holomorphic transformation on $T$ satisfying $s \circ A=s$, and observe that $s \circ I \circ A \circ I=s$. Since $I \circ A \circ I \neq I d$, the uniqueness of $A$ gives $I \circ A \circ I=A$. As $A(P)=I(P)$, the proof is complete. Q.E.D.

In what follows, we will assume that:

- $\bar{M}$ has genus $k \geq 1$.

- $I$ is an antiholomorphic involution without fixed points on $\bar{M}$.

- $A$ is a holomorphic involution with $2 k+2$ fixed points satisfying $A \circ I=I \circ A$. The last equality can be omitted if $k>1$ (it automatically holds).

- $A \circ I$ has some fixed points on $\bar{M}$ (hence infinitely many because $A \circ I$ is antiholomorphic). This condition can be omitted if $k$ is even (it automatically holds, see $[\mathrm{R}]$ ).

Label as $s$ a degree two meromorphic function on $\bar{M}$ satisfying $s \circ A=s$. If $k \geq 2, \bar{s} \circ I$ is $s$ followed by a Möbius transformation, and the fact $I \circ I=\mathrm{Id}$ 
gives, up to a suitable change of parameter, $s \circ I=-\bar{s}$. A degree two meromorphic function $s$ satisfying the last equation is not unique: for, observe that the changes $s \rightarrow\left(r_{1} s+i r_{2}\right) /\left(i r_{3} s+r_{4}\right), r_{i} \in \mathbb{R}, i=1,2,3,4$, yield a real three-parameter family of such functions. If $k=1$, we can use the ideas of Proposition 2.1 to obtain the same conclusions.

Write $\left\{Q_{1}, \ldots, Q_{k+1}, I\left(Q_{1}\right), \ldots, I\left(Q_{k+1}\right)\right\}$, the fixed point set of $A$, and label $d_{i}=s\left(Q_{i}\right), i=1, \ldots, k+1$. It is well known that there exists a meromorphic function $v$ on $\bar{M}$ of degree $k+1$ satisfying

$$
v^{2}=\prod_{i=1}^{k+1}\left(\frac{s-d_{i}}{s+\bar{d}_{i}}\right),
$$

Since $I$ has no fixed points, then $v \circ I=-1 / \bar{v}$.

Thus, $\bar{M}$ is conformally equivalent to the algebraic curve

$$
\left\{(s, v) \in(\mathbb{C} \cup\{\infty\})^{2}: v^{2}=\prod_{i=1}^{k+1}\left(\frac{s-d_{i}}{s+\bar{d}_{i}}\right)\right\}
$$

with the natural complex structure, and $I, A$ become

$$
I(s, v)=(-\bar{s},-1 / \bar{v}), \quad A(s, v)=(s,-v) .
$$

It is clear that $d_{i} \neq d_{j}, i \neq j$, and $d_{i} \neq-\bar{d}_{j}, i, j \in\{1, \ldots, k+1\}$.

For the sake of simplicity, we write $d_{i},-\bar{d}_{i}$ instead of $\left(d_{i}, 0\right),\left(-\bar{d}_{i}, \infty\right)$, respectively, $i=1, \ldots, k+1$. Write also $\infty_{1}=(\infty, 1), \infty_{2}=(\infty,-1)$.

Later we will need the following topological remarks. Let $\left\{\gamma_{1}, \ldots, \gamma_{k}, \chi_{1}, \ldots, \chi_{k}\right\}$ be a homology basis of $\bar{M}$ satisfying:

$$
I^{*}\left(\gamma_{i}\right)=\gamma_{i}, \quad I^{*}\left(\chi_{i}\right)=-\chi_{i}, \quad i=1, \ldots, k,
$$

and take any meromorphic 1-form $\omega$ verifying $I^{*}(\omega)=\bar{\omega}$.

Then, observe that

$$
\int_{\gamma_{i}} \omega=\int_{I^{*}\left(\gamma_{i}\right)} I^{*}(\omega)=\int_{\gamma_{i}} \bar{\omega},
$$

and analogously

$$
\int_{\chi_{i}} \omega=-\int_{\chi_{i}} \bar{\omega}, \quad i \in\{1, \ldots, k\} .
$$

Moreover, suppose that $[\omega]_{\infty}=P^{n} I(P)^{n}$ and take a conformal disk $D$ in $\bar{M}$ such that $P \in D, I(P) \notin D$. Then, if $\gamma$ is a closed curve winding once around $P$ and contained in $D$,

$$
\int_{\gamma} \omega=\int_{I^{*}(\gamma)} \bar{\omega}
$$

Since $I$ reverses the orientation, $\bar{M}$ is compact and $\omega$ has singularities only at $P$ and $I(P)$, general theory gives

$$
\int_{\gamma} \omega-\int_{I^{*}(\gamma)} \omega=0
$$

and thus

$$
\int_{\gamma} \omega=\int_{\gamma} \bar{\omega}
$$


We summarize these facts in the following remark:

Remark 2.1. For any meromorphic 1-form $\omega$ in $\bar{M}$ satisfying $I^{*}(\omega)=\bar{\omega}$,

$$
\int_{\gamma_{i}} \omega \in \mathbb{R}, \quad \int_{\chi_{i}} \omega \in i \mathbb{R}, \quad i=1, \ldots, k .
$$

Moreover, if $[\omega]_{\infty}=P^{n} I(P)^{n}$ then Residue $[\omega, P] \in i \mathbb{R}$.

We are interested in the meromorphic functions on $\bar{M}$ of degree $k+3$ satisfying $g \cdot I=-1 / \bar{g}$.

Proposition 2.2. Suppose $g$ is a meromorphic function on $\bar{M}$ of degree $k+3$ satisfying $g \circ I=-1 / \bar{g}$. Then, up to rigid motions in $\mathbb{C} \cup\{\infty\}=\mathbb{S}^{2}$ :

$$
g=\frac{s-\bar{\alpha}}{s+\alpha} h
$$

where $\alpha \in \mathbb{C}, \mathfrak{R e}(\alpha) \neq 0$ and $h$ is a meromorphic function on $\bar{M}$ of degree $k+1$ satisfying $h \circ I=-1 / \bar{h}$.

Proof. Up to a holomorphic rigid motion in $\mathbb{C} \cup\{\infty\}$ given by

$$
g \rightarrow(\lambda g+\mu) /(\bar{\mu} g-\bar{\lambda})
$$

for suitable $\lambda, \mu \in \mathbb{C}$, we can suppose $g$ has $k+3$ simple poles. Moreover, from our assumptions below the demonstration of Proposition 2.1, $A \cdot I$ has infinitely many fixed points. These facts, together with $g \circ I=-1 / \bar{g}$, yield $g \circ A \neq g$. For, observe that any fixed point $Q$ of $A \circ I$ satisfies $g(I(Q))=g(A(Q))$.

So, a suitable choice of $\lambda, \mu$ yields that $g-g \circ A$ has degree $2(k+3)$. Since $A$ has only $2 k+2$ fixed points, we can take $Q \in \bar{M}$ satisfying $[g-g(Q)]_{0} \geq Q A(Q)$. Label $\bar{\alpha}=s(Q)$. Up to a holomorphic rigid motion once again, we can assume that $g(Q)=g(A(Q))=0$ and $g(I(Q))=g(A(I(Q)))=\infty$. Thus, the meromorphic function $h=g(s+\alpha) /(s-\bar{\alpha})$ has degree $k+1$ and $h \circ I=-1 / \bar{h}$. Q.E.D.

Proposition 2.3. Suppose $h$ is a meromorphic function on $\bar{M}$ of degree $k+1$ satisfying $h \cdot I=-1 / \bar{h}$ and $A\left([h]_{0}\right) \sim[h]_{0}$. Then, up to relabelling of the Weierstrass point set of $\bar{M},\left\{d_{1}, \ldots, d_{k+1},-\bar{d}_{1}, \ldots,-\bar{d}_{k+1}\right\}$, there exist $\lambda, \mu \in \mathbb{C}$ such that

$$
h=\frac{\bar{\lambda} v+\bar{\mu}}{\lambda-\mu v} \text {. }
$$

Proof. Take a meromorphic function $f$ satisfying $[f]=[h]_{0} / A\left([h]_{0}\right)$ and $f \cdot A=1 / f$. Assume that there exists a point $Q \in \bar{M}$ such that $[h]_{0} \geq Q A(Q)$. If we write $h_{1}=h(s+\bar{s}(Q)) /(s-s(Q)), h_{1}$ has degree less than $k+1$ and satisfies $h_{1} \cdot I=-1 / \bar{h}_{1}$. The theory of hyperelliptic surfaces implies that $h_{1}$ is a rational function of $s$ (see [FK, p. 108], and thus $h_{1} \cdot I=1 / \bar{h}_{1}$, a contradiction.

Now, we can deduce that $f$ has degree either $k+1$ or zero. If not, as above, $f$ is a nontrivial rational function of $s$, and so, we can find a point $Q$ such that $[h]_{0} \geq Q A(Q)$ and get a contradiction.

The case $f=$ constant, i.e. $[h]_{0}=A\left([h]_{0}\right)$, implies, up to relabelling of the set $\left\{d_{1}, \ldots, d_{k+1},-\bar{d}_{1}, \ldots,-\bar{d}_{k+1}\right\}$, that $h=\mu v,|\mu|=1$, and we are done. For, observe that the nonexistence of any point $Q \in \bar{M}$ satisfying $[h]_{0} \geq Q A(Q)$ yields that $h$ has only simple zeroes and all of them are fixed points of $A$.

Therefore, we can suppose that $f$ has degree $k+1$ and, in particular, $1 / f=$ $f \cdot A \neq f$. The theory of compact Riemann surfaces (see [FK]) implies the existence 
of three polynomial functions $P_{0}, P_{1}, P_{2}$ of degree at most $k+1$ (and at least one of them of degree $k+1$ ) satisfying

$$
P_{2}(s) f^{2}+P_{1}(s) f+P_{0}=0 .
$$

Since $f \cdot A \neq f, f$ is not a rational function of $s$. As a consequence, $P_{1}^{2}(s)-$ $4 P_{2}(s) P_{0}(s)=\lambda^{2} w^{2}$, where $w=v\left(\prod_{i=1}^{k+1}\left(s+\bar{d}_{i}\right)\right)$, and defining $R_{1}=-\left(P_{1}+2 P_{0}\right) / \lambda$, $R_{2}=\left(P_{1}+2 P_{2}\right) / \lambda$ we deduce that

$$
f=\left(R_{1}(s)+w\right) /\left(R_{2}(s)+w\right) .
$$

On the other hand, $f \cdot A=1 / f$ and $w \circ A=-w$ give $R_{1}=-R_{2}$. Since $R_{1} \neq 0$ ( $f$ is not constant), we conclude that $u=w / R_{1}(s)$ has degree $k+1$. Therefore, the zeroes and poles of $u$ are fixed points of $A$ (see [FK, p. 108]), and we have $[u]_{0}[u]_{\infty}=[w]_{0}$.

Since $[1+u]_{0}=[f]_{0}=[h]_{0}$, we obtain $[(1+u) /(1+\bar{u} \cdot I)]=[h] I\left([u]_{\infty}\right) /[u]_{\infty}$, and so there exists a meromorphic function $u_{1}$ satisfying $\left[u_{1}\right]=I\left([u]_{\infty}\right) /[u]_{\infty}$.

The case $[u]_{\infty}=I\left([u]_{\infty}\right)$ implies that $u_{1}$ is a constant and $u \cdot I=\theta \bar{u},|\theta|=1$. Thus, $h=\mu(1+u) /(1+\bar{\theta} u),|\mu|=1$, and so $h \cdot I=1 / \bar{h}$, a contradiction. Therefore, we can suppose $u_{1}$ is not constant.

As $u_{1}$ has simple poles and zeroes and all of them are fixed points of $A$, we can deduce (see [FK, p. 108]) that $u_{1}$ has degree $k+1$ and $I\left(\left[u_{1}\right]_{\infty}\right)\left[u_{1}\right]_{\infty}=[w]_{0}$. In particular, $[u]_{0}=I\left([u]_{\infty}\right)$ and $u_{1}=c u, c \neq 0$. Hence, up to relabelling of the set $\left\{d_{1}, \ldots, d_{k+1},-\bar{d}_{1}, \ldots,-\bar{d}_{k+1}\right\}, u=\lambda v, \lambda \neq 0$, and therefore $h=\theta(1+\lambda v) /(\bar{\lambda}-v)$, $|\theta|=1$. Q.E.D.

Lemma 2.1. For $k=\operatorname{Genus}(\bar{M})$ even, let $h$ be a meromorphic function on $\bar{M}$ of degree $k+1$ and take $P \in \bar{M}$ such that $P \neq A(P), A(P) \neq I(P)$. Suppose $h \circ I=-1 / \bar{h}$ and $D^{2}[h]_{0} \sim A\left(D^{2}[h]_{0}\right)$, where $D=P I(P) \in \operatorname{Div}(\bar{M})$.

If there exists $J \in \operatorname{Aut}(\bar{M})$ of order $p>1$ satisfying $J \circ I=I \circ J, J(P)=P$ and $J\left([h]_{0}\right)=[h]_{0}$, then $p$ divides $k+1$ and either $[h]_{0} \geq A(P)^{2} I(P)^{p-2}$ or $[h]_{0} \geq$ $A(I(P))^{2} P^{p-2}$.

Proof. Since $A(P) \neq I(P), s(P) \notin i \mathbb{R}$. Define $z=(s-s(P)) /(s+\bar{s}(P))$ and $e_{i}=z\left(d_{i}\right), i=1, \ldots, k+1$. Observe that $\left|e_{i}\right| \neq 1$. Then, up to biholomorphisms,

$$
\begin{gathered}
\bar{M}=\left\{(z, x) \in(\mathbb{C} \cup\{\infty\})^{2}: x^{2}=\prod_{i=1}^{k+1}\left(\frac{z-e_{i}}{\bar{e}_{i} z-1}\right)\right\}, \\
I(z, x)=(1 / \bar{z},-1 / \bar{x}), \quad A(z, x)=(z,-x) .
\end{gathered}
$$

As usual, $e_{i}=\left(e_{i}, 0\right), 1 / \bar{e}_{i}=\left(1 / \bar{e}_{i}, \infty\right)$, for $i=1, \ldots, k+1$, and

$$
0_{j}=\left(0,(-1)^{j}\left(\prod_{i=1}^{k+1} e_{i}\right)^{1 / 2}\right), \quad \infty_{j}=I\left(0_{j}\right), \quad j=1,2 .
$$

For suitable choice of the branch, $P=0_{1}, A(P)=0_{2}, I(P)=\infty_{1}$ and $I(A(P))=$ $\infty_{2}$.

It is clear that $A \circ J \circ A=J$ (see [FK, p. 108]), and so $z \circ J=L \circ z$, where $L$ is a Möbius transformation. As $z(P)=0, z(I(P))=\infty$ and $J(P)=P$, we deduce that $z \circ J=\theta z$, where $\theta^{p}=1$. But $P \neq A(P)$ and $J(D)=D$ give $J^{i} \neq A, i \in\{1, \ldots, p\}$, and therefore without loss of generality $\theta=\operatorname{Exp}(2 \pi i / p)$. Up to relabelling of the set $\left\{e_{1}, \ldots, e_{k+1}, 1 / \bar{e}_{1}, \ldots, 1 / \bar{e}_{k+1}\right\}, x^{2} \circ J=x^{2}$ and thus $p$ divides $k+1$. In particular, $p$ is odd and $J(z, x)=(\theta z, x)$. 
Note also that $\{P, A(P), I(P), I(A(P))\}$ is the fixed point set of $J^{i}, i \in\{1, \ldots$, $p-1\}$.

Denote by $f$ a meromorphic function satisfying $[f]=D^{2}[h]_{0} / A\left(D^{2}[h]_{0}\right)$ and $f \circ A=1 / f$. It is clear that $f \circ J=\lambda f, \lambda^{p}=1$. But $J \circ A=A \circ J$ gives $\lambda^{2}=1$ and so $\lambda=1$ ( $p$ is odd!).

First, observe that $f$ is not constant. For, note that in this case our hypothesis in the lemma implies $[h]_{0} \geq A(D)$ and so $[h]_{\infty}=I\left([h]_{0}\right) \geq I(A(D))=A(D)$. Thus, $h$ has degree less than $k+1$, a contradiction.

In particular, $1 / f=f \circ A \neq f$. As a consequence, the theory of hyperelliptic surfaces (see [FK, p. 108]) implies that $f$ has degree greater than $k$. In fact, $n=\operatorname{Degree}(f)=k+i, i \in\{1,2,3,4,5\}$.

On the other hand, $f \circ J=f$ means that $n=p q, q \in \mathbb{N}$. Since $p$ divides $k+1$, $p \geq 5$ yields $n=k+1$.

When $p=3, n=k+1$ too. In other cases, $n=k+4$. Therefore, there exists a unique $Q \in \bar{M}$ such that $D[h]_{0}, A\left(D[h]_{0}\right) \geq Q$. If $Q \notin\{P, A(P), I(P), I(A(P))\}$, then $Q \neq J(Q)$ and $D[h]_{0}, A\left(D[h]_{0}\right) \geq J(Q)$, which is absurd. Therefore, without lost of generality we can suppose $Q=P$. Since $D \neq A(D)$, we deduce that $A\left([h]_{0}\right) \geq P$ and hence $D[h]_{0}, A\left(D[h]_{0}\right) \geq P A(P)$, a contradiction again. Hence, $n=k+1$.

Then, there are exactly four points $Q_{1}, i=1,2,3,4$ (counting multiplicities), in $\bar{M}$ satisfying $D[h]_{0}, A\left(D[h]_{0}\right) \geq Q_{i}, i=1,2,3,4$. Label as $E$ the integral divisor of degree four determined by these points. Since $f \circ A=1 / f$ and $f \circ J=f$, we have $A(E)=J(E)=E$. So, it is not hard to prove that $Q_{i} \in\{P, A(P), I(P), I(A(P))\}$, $i \in\{1,2,3,4\}$. If $E=P A(P) I(P) I(A(P))$, then $[h]_{0} \geq A(P) I(A(P))$ and therefore $[h]_{\infty}=I\left([h]_{0}\right) \geq A(P) A(I(P))$. We deduce that $h$ has degree less than $k$, a contradiction.

Without loss of generality, we can suppose $E=P^{2} A(P)^{2}$, and thus $[h]_{0} \geq A(P)^{2}$, $[f]_{0}=[h]_{0} I(P)^{2} / A(P)^{2}$. Furthermore, $f \circ J=f$ gives $[f]_{0} \geq I(P)^{p}$ and so $[h]_{0} \geq$ $I(P)^{p-2}$. This concludes the proof. Q.E.D.

Lemma 2.2. Take $P \in \bar{M}$ such that $P=A(P)$ and $J \in \operatorname{Aut}(\bar{M})$ an automorphism of order $p>1$ satisfying $J \circ I=I \circ J, J(P)=P$. Let $h$ be a meromorphic function of degree $k+1$ satisfying $h \cdot I=-1 / \bar{h},[h]_{0} \sim A\left([h]_{0}\right)$ and $J\left([h]_{0}\right)=[h]_{0}$. Then $p$ divides $k$ and, up to a biholomorphism and relabelling of the Weierstrass point set of $\bar{M}$,

$$
\begin{gathered}
\bar{M}=\left\{(z, x) \in(\mathbb{C} \cup\{\infty\})^{2}: x^{2}=z \prod_{i=1}^{k / p}\left(\left(z^{p}-e_{i}^{p}\right) /\left(\bar{e}_{i}^{p} z^{p}-1\right)\right)\right\}, \\
I(z, x)=(1 / \bar{z},-1 / \bar{x}), \quad A(z, x)=(z,-x), \quad J(z, x)=\left(\theta^{2} z, \theta x\right), \quad h=\mu x,
\end{gathered}
$$

where $e_{i}, \mu \in \mathbb{C}, i=1, \ldots, k,\left|e_{i}\right| \neq 1,|\mu|=1$ and $\theta^{p}=1$.

Proof. We suppose $P=d_{k+1}$. Since $A(P) \neq I(P), s(P) \notin i \mathbb{R}$. Define $z=$ $(s-s(P)) /(s+\bar{s}(P))$ and $e_{i}=z\left(d_{i}\right), i=1, \ldots, k$. Observe that $\left|e_{i}\right| \neq 1$. Then, up to biholomorphisms,

$$
\begin{gathered}
\bar{M}=\left\{(z, x) \in(\mathbb{C} \cup\{\infty\})^{2}: x^{2}=z \prod_{i=1}^{k}\left(\frac{z-e_{i}}{\bar{e}_{i} z-1}\right)\right\}, \\
I(z, x)=(1 / \bar{z},-1 / \bar{x}), \quad A(z, x)=(z,-x) .
\end{gathered}
$$


As usual, $e_{i}=\left(e_{i}, 0\right), 1 / \bar{e}_{i}=\left(1 / \bar{e}_{i}, \infty\right)$, for $i=1, \ldots, k$, and $0=(0,0), \infty=$ $(\infty, \infty)$. It is clear that $P=0, I(P)=\infty$.

As in Lemma 2.1, $z \circ J=\xi z$, where $\xi^{p}=1$. Moreover, $J \circ A=A \circ J$ yields, up to relabelling of the set $\left\{e_{1}, \ldots, e_{k}, 1 / \bar{e}_{1}, \ldots, 1 / \bar{e}_{k}\right\}, x^{2} \circ J=\xi x^{2}$ and $J(z, x)=$ $\left(\xi z, \xi^{1 / 2} x\right)$. Then, $k=p q$, where $q \in \mathbb{N}$, and $\left(\xi^{1 / 2}\right)^{p}=1$. In particular, $p$ even gives $J^{p / 2}=A$.

Suppose there exists a meromorphic function $h$ satisfying the hypothesis of the lemma. Up to change of parameters and relabellings, we only need to prove that $h=\mu x,|\mu|=1$. Label as $f$ a meromorphic function satisfying $[f]=[h]_{0} / A\left([h]_{0}\right)$ and $f \circ A=1 / f$. It is clear that $f \circ J=\lambda f, \lambda^{p}=1$. Furthermore, $J \circ A=A \circ J$ gives $\lambda^{2}=1$. If $p$ is even, then the above expression for $J$ gives $J^{p / 2}=A$, which ensures that $f \cdot J^{p / 2}=\lambda^{p / 2} f=1 / f$, and so $f$ is constant. Since there are no points $Q \in \bar{M}$ such that $[h]_{0} \geq Q A(Q)$ (see the proof of Proposition 2.3), all the zeroes and poles of $h$ are Weierstrass points of $\bar{M}$. Therefore, up to relabelling of the set $\left\{e_{1}, \ldots, e_{k}, 1 / \bar{e}_{1}, \ldots, 1 / \bar{e}_{k}, 0, \infty\right\}, h=\mu x,|\mu|=1$ and we are done. If $p$ is odd, then $\lambda=1$ and $f \cdot J=f$. In particular, $p$ divides the degree of $f$. Since degree $(f) \leq k+1$ and $p$ divides $k$, we have degree $(f) \leq k$ and so $f$ is a rational function of $s$. As above, there are no points $Q \in \bar{M}$ such that $[h]_{0} \geq Q A(Q)$, and hence $f$ is constant, that is, as before, $h=\mu x,|\mu|=1$. Q.E.D.

Lemma 2.3. For arbitrary genus $k \geq 1$, take $P \in \bar{M}$ such that $I(P)=A(P)$.

Let $h$ be a meromorphic function of $\bar{M}$ with degree $k+1$ and verifying $h \circ I=$ $-1 / \bar{h}$.

If there exists $J \in \operatorname{Aut}(\bar{M})$ of order $p>1$ satisfying $J \circ I=I \circ J, J(P I(P))=$ $P I(P)$ and $J\left([h]_{0}\right)=[h]_{0}$, then $J$ is either $A, B$ or $A \cdot B$, where $A, B$ and $A \cdot B$ are the only holomorphic involutions on $\bar{M}$ leaving invariant the integral divisor $P I(P)$.

Proof. Without loss of generality, $s(P)=s(I(P))=\infty$. It is clear that $s \cdot J=\lambda s+\mu$, $\lambda, \mu \in \mathbb{C}$. As $J \cdot I=I \cdot J$, then $\lambda \in \mathbb{R}, \mu \in i \mathbb{R}$. Moreover, $J^{p}=\mathrm{Id}$ gives $\lambda^{p}=1$ and so either $\lambda=1$ (arbitrary $p$ ) or $\lambda=-1$ ( $p$ even). If $\lambda=1, J^{p}=$ Id gives $\mu=0$ and so $J=A$.

Suppose $\lambda=-1$. In particular, $p$ is even. Up to the change of parameter $s \rightarrow s-\mu / 2$, we can assume that $s \cdot J=-s$. Define $w=\left(\prod_{i=1}^{k+1}\left(s+\bar{d}_{i}\right)\right) v$. Since $J \cdot A=A \cdot J$ (see [FK, p. 108]), $J$ leaves the fixed point set of $A$ invariant, and so $w^{2} \cdot J=w^{2}$, that is, $w^{2}(-s)=w^{2}(s)$. Therefore, either $w \cdot J=w$ or $w \cdot J=-w$. So, either $J(s, w)=(-s, w)$ or $J(s, w)=(-s,-w)$. This concludes the proof. Q.E.D.

\section{Minimal Klein bottles with total Curvature $-8 \pi$. THE SYMMETRY GROUP OF NONORIENTABLE MINIMAL SURFACES OF HIGH GENUS AND CRITICAL TOTAL CURVATURE}

Let $N$ be a conformal nonorientable noncompact surface. Let $x: N \rightarrow \mathbb{R}^{3}$ denote a complete conformal minimal immersion with finite total curvature $C(N)$ of $N$ in three dimensional Euclidean space.

Label $\pi_{0}: M \rightarrow N$ and $I: M \rightarrow M$ the orientable two sheeted cover of $N$ and the antiholomorphic two deck transformation associated to this cover, respectively. By results of Huber and Meeks mentioned in Section 1, $N$ is conformally diffeomorphic to a nonorientable compact surface $\bar{N}$ punctured in a finite set of 
points $\left\{\bar{P}_{1}, \ldots, \bar{P}_{r}\right\}$, and the Euler characteristic $\chi(\bar{N})$ of $\bar{N}$ and $C(N) /(2 \pi)$ are congruent modulo 2 .

Assume that $\chi(\bar{N}) \leq 1-k, k \geq 1$, and the degree of the generalized Gauss map $G$ is $k+3$. Note that in the case $k=1$, Degree $(G)=4$ and the congruence formula of Meeks implies in fact $\chi(\bar{N}) \leq 0$. The Jorge-Meeks formula (1.3) and the congruence formula of Meeks yield the following possibilities:

(i) $\chi(\bar{N})=1-k, r=1$ and $\nu_{1}=3$,

(ii) $\chi(\bar{N})=1-k, r=2$ and $\nu_{1}=\nu_{2}=1$,

(iii) $\chi(\bar{N})=-1-k, r=1$ and $\nu_{1}=1$.

Since $x(N)$ is not a plane, (iii) is impossible (see [JM]). A nonexistence theorem in [K1] ensures that (ii) does not happen either. So, $\chi(\bar{N})=1-k, N=\bar{N}-\left\{\bar{P}_{1}\right\}$, $\bar{P}_{1} \in \bar{N}$, and $\nu_{1}=3$.

In other words, $x_{0}=x \cdot \pi_{0}$ is a complete minimal immersion with total curvature $-4(3+k) \pi$ of $M$ in $\mathbb{R}^{3}$ satisfying $x_{0} \cdot I=x_{0}$. We will use the same notation $I$ for the unique conformal extension of the antiholomorphic involution in $M$ to the two sheeted orientable cover $\bar{M}$ of $\bar{N}$. Denote this cover also by $\pi_{0}$, and observe that $I$ has no fixed points on $\bar{M}$.

If we write $P_{1} \in \bar{M}$ for a lift of $\bar{P}_{1}$, then $M=\bar{M}-\left\{P_{1}, P_{2}=I\left(P_{1}\right)\right\}$, and $\nu_{1}=3$.

For the remainder of this section, we will assume that $\bar{M}$ is hyperelliptic. As we have mentioned in Section 2, up to holomorphic transformations,

$$
\begin{gathered}
\bar{M}=\left\{(s, v) \in(\mathbb{C} \cup\{\infty\})^{2}: v^{2}=\prod_{i=1}^{k+1}\left(\left(s-d_{i}\right) /\left(s+\bar{d}_{i}\right)\right)\right\}, \\
I(s, v)=(-\bar{s},-1 / \bar{v}) .
\end{gathered}
$$

Remember that the points $d_{i},-\bar{d}_{i}, i=1, \ldots, 1+k$, satisfy the algebraic condition of nondegeneration:

$$
d_{i} \notin i \mathbb{R}, \quad d_{i} \neq d_{j}, \quad d_{i} \neq-\bar{d}_{j}, \quad i \neq j, \quad \text { for } i, j \in\{1, \ldots, 1+k\} .
$$

Defining $w=v \prod_{i=1}^{k+1}\left(s+\bar{d}_{i}\right)$ and $P(s)=w^{2}$, this condition means that

$$
\text { Resultant }\left[P(s), P^{\prime}(s), s\right] \neq 0 .
$$

In the case $k=1$, labelling $x_{1}=\mathfrak{R e}\left(d_{1}+d_{2}\right), y_{1}=\mathfrak{T} \mathfrak{m}\left(d_{1}+d_{2}\right), x_{2}=\mathfrak{R e}\left(d_{1} d_{2}\right)$ and $y_{2}=\mathfrak{T} \mathfrak{m}\left(d_{1} d_{2}\right)$, it can be written as follows:

$$
\left(\left(4 y_{2}-2 x_{1} y_{1}\right)^{2}+\left(4 x_{2}+y_{1}^{2}-x_{1}^{2}\right)^{2}\right)\left(x_{1}^{2} x_{2}+x_{1} y_{1} y_{2}-y_{2}^{2}\right)^{2} \neq 0 .
$$

Call $A$ the holomorphic involution on $\bar{M}$ fixing $d_{i},-\bar{d}_{i}, i=1, \ldots, 1+k$, given by $A(s, v)=(s,-v)$.

As usual, write $(g, \eta)$ for the Weierstrass data of $x_{0}$ and $\phi_{j}, j=1,2,3$, for the 1-forms given in (3.1). By Osserman's theorem (see Section 1), $(g, \eta)$ extends meromorphically to $\bar{M}$. Proposition 2.2 implies that $g=h(s-\bar{\alpha}) /(s+\alpha)$, where $\alpha \notin i \mathbb{R}, h$ has degree $1+k$ and $h \cdot I=-1 / \bar{h}$. On the other hand, taking into account that $\nu_{1}=3$ in (1.4) and (1.1), we have

$$
[\eta w h /((s-\bar{\alpha})(s+\alpha) d s)]=[h]_{0}^{2} /\left(\infty_{1}^{k-3} \infty_{2}^{k-3} P_{1}^{4} I\left(P_{1}\right)^{4}\right) .
$$

Therefore, it is not hard to deduce that

$$
[h]_{0} D^{2} \sim A\left([h]_{0} D^{2}\right)
$$

where $D=A\left(P_{1}\right) A\left(I\left(P_{1}\right)\right)$. 
Suppose $A\left(P_{1}\right)=I\left(P_{1}\right)$, that is, up to a natural change of variables, $P_{1}=\infty_{1}$, $P_{2}=I\left(P_{1}\right)=\infty_{2}$. Remember that in the case $k=1$, Proposition 2.1 yields in fact the existence of a holomorphic involution $A$ with four fixed points satisfying $A \cdot I=I \cdot A, A\left(P_{1}\right)=I\left(P_{1}\right)$, and thus we can omit this assumption. In what follows, up to the change $s \rightarrow s+i \mathfrak{T} \mathfrak{m}(\alpha)$, we will assume that $\alpha \in \mathbb{R}$. Up to rigid motions, Proposition 2.3 yields $h=(v-\bar{\lambda}) /(\lambda v+1)$, where $\lambda \in \mathbb{C}$, and thus (1.1), (1.2) and (1.4) imply that:

$$
g=\frac{s-\alpha}{s+\alpha} \frac{v-\bar{\lambda}}{\lambda v+1}, \quad \eta g=i\left(s^{2}-\alpha^{2}\right)\left(\lambda v-\bar{\lambda} / v+1-|\lambda|^{2}\right) d s .
$$

From the algebraic point of view, bilinear relations of Riemann and residues concerning $\phi_{j}, j=1,2,3$, can be easily interpreted computing them from the meromorphic data $\left(g^{\prime}, \eta^{\prime}\right)$ of minimal immersions $R \cdot x_{0}$ with vertical normal vectors at both ends $P_{1}, I\left(P_{1}\right)$ ( $R$ is a suitable rigid motion in $\left.\mathbb{R}\right)$. This is the aim of the following lemma:

Lemma 3.1. On the assumption that $P_{1}=\infty_{1}$ and $P_{2}=I\left(P_{1}\right)=\infty_{2}$, there exist a rigid motion $R$ and a homothety $H$ in $\mathbb{R}^{3}$ such that the Weierstrass representation $\left(g^{\prime}, \eta^{\prime}\right)$ of $H \cdot R \cdot x_{0}$ is given by

$$
\begin{gathered}
g^{\prime}=\frac{(s+\beta-Y) v-(s-\beta-\bar{Y})}{(s+\beta+Y) v+(s-\beta+\bar{Y})}, \\
\eta^{\prime} g^{\prime}=i\left(\left((s-\beta)^{2}-\bar{Y}^{2}\right) / v-\left((s+\beta)^{2}-Y^{2}\right) v+4(i \mathfrak{T} \mathfrak{m}(Y) s-\mathfrak{R e}(Y) \beta)\right) d s,
\end{gathered}
$$

where $Y=2 \alpha \lambda /\left(|\lambda|^{2}+1\right), \beta=\alpha\left(|\lambda|^{2}-1\right) /\left(|\lambda|^{2}+1\right)$.

In particular, $|Y|^{2}+\beta^{2}=\alpha^{2} \neq 0$.

Proof. Pick a rigid motion $R$ in $\mathbb{R}^{3}$ such that

$$
g^{\prime}=R \cdot g=\frac{\lambda+1}{\bar{\lambda}+1} \frac{g-g\left(\infty_{1}\right)}{\bar{g}\left(\infty_{1}\right) g+1}=\frac{(s+\beta-Y) v-(s-\beta-\bar{Y})}{(s+\beta+Y) v+(s-\beta+\bar{Y})}
$$

where $\beta, Y$ are defined as in the lemma.

If $\left(\eta^{\prime}, g^{\prime}\right)$ is the Weierstrass data of $R \cdot x_{0}$, taking into account that $\nu_{1}=3,(1.1)$, (1.2), and (1.4) give us

$$
\eta^{\prime} g^{\prime}=-i a^{2}((s+\beta-Y) v-(s-\beta-\bar{Y}))((s+\beta+Y) v+(s-\beta+\bar{Y})) \frac{d s}{v}
$$

where $a \in \mathbb{R}-\{0\}$. This concludes the proof. Q.E.D.

Remark 3.1. From Lemma 3.1, if $P_{1}=\infty_{1}, P_{2}=\infty_{2}$ :

$$
\begin{gathered}
\eta^{\prime}=-i\left((s-\beta+\bar{Y})^{2} / v+(s+\beta+Y)^{2} v+2(s-\beta+\bar{Y})(s+\beta+Y)\right) d s, \\
\eta^{\prime} g^{\prime 2}=-i\left((s+\beta-Y)^{2} v+(s-\beta-\bar{Y})^{2} / v-2(s+\beta-Y)(s-\beta-\bar{Y})\right) d s .
\end{gathered}
$$

Take the meromorphic functions on $\bar{M}$ given by

$$
\begin{gathered}
f_{1}=(v+1 / v), \quad f_{2}=(v-1 / v), \\
f_{3}=s(v+1 / v), \quad f_{4}=s(v-1 / v), \quad f_{5}=s^{2}(v+1 / v) .
\end{gathered}
$$

Fix our attention on the case $k=1$, that is, when $\bar{N}$ is a conformal Klein Bottle $K$, and $\bar{M}$ is a conformal torus $T$.

We will use once again the notation $x_{1}=\mathfrak{R e}\left(d_{1}+d_{2}\right), y_{1}=\mathfrak{T} \mathfrak{m}\left(d_{1}+d_{2}\right)$, $x_{2}=\mathfrak{R e}\left(d_{1} d_{2}\right)$ and $y_{2}=\mathfrak{T} \mathfrak{m}\left(d_{1} d_{2}\right)$, and label $x_{3}=\mathfrak{R e}(Y), y_{3}=\mathfrak{T} \mathfrak{m}(Y)$. From 
Proposition 2.1 and without loss of generality $P_{i}=\infty_{i}, i=1,2$. For technical reasons, we need the following lemma:

Lemma 3.2. If $k=1$ and we define $\psi_{1}=\frac{1}{2}\left(\eta^{\prime}-\eta^{\prime} g^{2}\right), \psi_{2}=\frac{i}{2}\left(\eta^{\prime}+\eta^{\prime} g^{\prime 2}\right)$ and $\psi_{3}=\eta^{\prime} g^{\prime}$ as in (1.1), then $\psi_{i} \approx \tau_{i}, i=1,2,3$, where

$$
\begin{aligned}
\tau_{1}= & \left(\left(x_{3} y_{1}+2 \beta y_{3}\right) f_{1}+i x_{3}\left(x_{1}-2 \beta\right) f_{2}+2 y_{3} f_{4}\right) d s, \\
\tau_{2}= & \left(\left(\beta^{2}+x_{3}^{2}-y_{3}^{2}-\left(x_{2}+y_{1}^{2}\right) / 3\right) f_{1}\right. \\
& \left.+i\left(2 x_{3} y_{3}+\left(y_{2}-x_{1} y_{1}\right) / 3\right) f_{2}+\left(2 \beta-2 x_{1} / 3\right) f_{4}\right) d s, \\
\tau_{3}= & \left(\left(\beta y_{1}+y_{2}-2 x_{3} y_{3}-x_{1} y_{1} / 2\right) f_{1}\right. \\
& \left.+i\left(\beta x_{1}-\beta^{2}+x_{2}+x_{3}^{2}-y_{3}^{2}-x_{1}^{2} / 2\right) f_{2}+y_{1} f_{4}\right) d s .
\end{aligned}
$$

Proof. Defining as above $w=v\left(s+\bar{d}_{1}\right)\left(s+\bar{d}_{2}\right)$, it is straightforward to check that

$$
\psi_{i}-\tau_{i}=d\left(h_{i}\right), \quad i=1,2,3,
$$

where

$$
\begin{gathered}
d\left(h_{1}\right)=d\left(-2 i x_{3} w\right)-i((s-\beta+\bar{Y})(s+\beta+Y)+(s+\beta-Y)(s-\beta-\bar{Y})) d s, \\
d\left(h_{2}\right)=d\left(2\left(s+i y_{1}\right) w / 3\right) \\
\quad+((s-\beta+\bar{Y})(s+\beta+Y)-(s+\beta-Y)(s-\beta-\bar{Y})) d s, \\
d\left(h_{3}\right)=d\left(i\left(x_{1}-2 \beta\right) w\right)+i(4(i \mathfrak{T} \mathfrak{m}(Y) s-\mathfrak{R e}(Y) \beta)) d s . \quad \text { Q.E.D. }
\end{gathered}
$$

Consider $\left\{\gamma_{1}, \chi_{1}\right\}$ a homology basis of $T$ satisfying: $I^{*}\left(\gamma_{1}\right)=\gamma_{1}, I^{*}\left(\chi_{1}\right)=-\chi_{1}$. Take also a positively oriented Jordan curve $\gamma$ lying in a conformal disc of $T$, winding once around $\infty_{1}$ and leaving $\infty_{2}$ at the outside.

Since $x_{0}$ is well defined, then for the 1 -forms $\psi_{j}, j=1,2,3$, have no real periods and so from Remark 2.1:

$$
\int_{\gamma_{1}} \psi_{j}=0, \quad j=1,2,3
$$

As a consequence, there exist suitable constants $\lambda_{i}, \mu_{i} \in \mathbb{C}, i=1,2,3$, such that

$$
\lambda_{1} \psi_{1} \approx \mu_{1} \psi_{2}, \quad \lambda_{2} \psi_{1} \approx \mu_{2} \psi_{3}, \quad \lambda_{3} \psi_{2} \approx \mu_{3} \psi_{3} .
$$

It is clear that each point $\left(x_{1}, x_{2}, x_{3}, y_{1}, y_{2}, y_{3}, \beta\right)$ of $\mathbb{R}^{7}$ satisfying $x_{3}^{2}+y_{3}^{2}+\beta^{2} \neq 0$ and (3.1) yields (defining $d_{1}+d_{2}=x_{1}+i y_{1}, d_{1} d_{2}=x_{2}+i y_{2}$ and $Y=x_{3}+i y_{3}$ ) a meromorphic data $\left(T, I, g^{\prime}, \eta^{\prime}\right)$ as in Lemma 3.1 that could appear associated to a minimal immersion of $K-\left\{\bar{P}_{1}\right\}$ in $\mathbb{R}^{3}$ with total curvature $-8 \pi$, and vice versa. (3.5):

The next lemma summarizes the algebraic information arising from (3.4) and

Lemma 3.3. Under the conditions (3.1) and $x_{3}^{2}+y_{3}^{2}+\beta^{2} \neq 0$, the equations (3.4) and (3.5) lead to the following algebraic subvarieties of $\mathbb{R}^{7}$ :

$$
\begin{gathered}
R_{1}=\left\{x_{2}=\left(2 \beta^{2}-2 \beta x_{1}+x_{1}^{2}\right) / 2, x_{3}=0, y_{1}=0, y_{2}=0, y_{3}=0\right\}, \\
R_{2}=\left\{x_{2}=x_{1}\left(3 x_{1}-2 \beta\right) / 8, x_{3}=0, y_{1}=0, y_{2}=0, y_{3}^{2}=\left(2 \beta-x_{1}\right)\left(x_{1}-4 \beta\right) / 8\right\}, \\
R_{3}=\left\{x_{2}=\left(2 \beta^{2}-2 \beta x_{1}+x_{1}^{2}\right) / 2, x_{3}=0, y_{1}^{2}=\left(x_{1}^{2}-8 \beta^{2}\right) / 2, y_{2}=x_{1} y_{1}, y_{3}=0\right\}, \\
S_{1}=\left\{x_{1}=2 \beta, x_{2}=\beta^{2}-x_{3}^{2}, y_{1}=0, y_{2}=0, y_{3}=0\right\},
\end{gathered}
$$




$$
\begin{gathered}
S_{2}=\left\{x_{1}=2 \beta, x_{2}=\left(4 \beta^{4}-\beta^{2} y_{1}^{2}-y_{1}^{4}\right) /\left(4 \beta^{2}+y_{1}^{2}\right),\right. \\
\left.x_{3}=0, y_{2}=4 \beta^{3} y_{1} /\left(4 \beta^{2}+y_{1}^{2}\right), y_{3}=0\right\}, \\
S_{3}=\left\{x_{1}=0, y_{1}=0, y_{2}^{2}=3 x_{3}^{4}+2 x_{3}^{2} x_{2}-x_{2}^{2}, y_{3}=0, \beta=0\right\} .
\end{gathered}
$$

Proof. From Lemma 3.2, (3.4) is equivalent to

$$
\text { Residue }\left[\tau_{3}, \infty_{1}\right]=-i\left(x_{1}\left(2 \beta^{2}-2 \beta x_{1}+x_{1}^{2}-2 x_{2}-2 x_{3}^{2}-2 y_{1}^{2}+2 y_{3}^{2}\right)+2 y_{1} y_{2}\right)=0 \text {, }
$$

$$
\begin{aligned}
& \text { Residue }\left[\eta^{\prime} g^{\prime 2}, \infty_{1}\right]=\text { Residue }\left[-\tau_{1}-i \tau_{2}, \infty_{1}\right] \\
&=2 i\left(2 \beta-x_{1}-2 i y_{3}\right)\left(x_{1}\left(x_{3}-i y_{1}\right)+i y_{2}\right)=0 .
\end{aligned}
$$

Assuming that $x_{1}=0$, then $2 y_{1} y_{2}=-2\left(\beta-i y_{3}\right) y_{2}=0$. Condition (3.1) gives $y_{2} \neq 0$, and so $y_{1}=\beta=y_{3}=0$. In this case, $\tau_{1}=0, \tau_{2}=\left(\left(3 x_{3}^{2}-x_{2}\right) f_{1}+i y_{2} f_{2}\right) / 3$ and $\tau_{3}=y_{2} f_{1}+i\left(x_{2}+x_{3}^{2}\right) f_{2}$. Since (3.5) is equivalent to $\lambda_{1} \tau_{1}=\mu_{1} \tau_{2}, \lambda_{2} \tau_{1}=\mu_{2} \tau_{3}$, $\lambda_{3} \tau_{2}=\mu_{3} \tau_{3}$, we deduce that $i\left(x_{2}^{2}-2 x_{2} x_{3}^{2}-3 x_{3}^{4}+y_{2}^{2}\right)=0$, which leads to $S_{3}$.

Suppose now $x_{1} \neq 0$. In this case, the above equations of residues again imply either

(i) $x_{3}=y_{2}-x_{1} y_{1}=2 \beta^{2}-2 \beta x_{1}+x_{1}^{2}-2 x_{2}+2 y_{3}^{2}=0$

or

(ii) $y_{3}=2 \beta-x_{1}=2 \beta^{3}-2 \beta x_{3}^{2}-2 \beta y_{1}^{2}+y_{1} y_{2}-2 \beta x_{2}=0$.

In the first case (i), $\tau_{1}=2 y_{3}\left(\beta f_{1}+f_{4}\right), \tau_{2}=f_{1}\left(4 \beta^{2}+2 \beta x_{1}-x_{1}^{2}-2 y_{1}^{2}-8 y_{3}^{2}\right) / 6+$ $2 f_{4}\left(3 \beta-x_{1}\right) / 3$ and $\tau_{3}=y_{1}\left(f_{4}+\left(2 \beta+x_{1}\right) f_{1} / 2\right)$. From (3.5), we have $\lambda_{1} \tau_{1}=\mu_{1} \tau_{2}$, $\lambda_{2} \tau_{1}=\mu_{2} \tau_{3}, \lambda_{3} \tau_{2}=\mu_{3} \tau_{3}$, and so

$$
y_{3}\left(8 \beta^{2}+x_{1}^{2}+2 y_{1}^{2}+8 y_{3}^{2}-6 \beta x_{1}\right) / 3=x_{1} y_{1} y_{3}=y_{1}\left(8 \beta^{2}-x_{1}^{2}+2 y_{1}^{2}+8 y_{3}^{2}\right) / 6=0 .
$$

As $x_{1} \neq 0$, we obtain $y_{1} y_{3}=0$. If $y_{3} \neq 0$, then $y_{1}=8 \beta^{2}+x_{1}^{2}+8 y_{3}^{2}-6 \beta x_{1}=0$, which together with (i) gets $R_{2}$. If $y_{1} \neq 0$, then $y_{3}=8 \beta^{2}-x_{1}^{2}+2 y_{1}^{2}=0$, and taking into account (i), it yields $R_{3}$. When $y_{3}=y_{1}=0$, (i) gives $R_{1}$.

In the second case (ii), $x_{1} \neq 0$ entails that $\beta \neq 0$, and defining $f=2 \beta f_{4}+$ $i\left(y_{2}-2 \beta y_{1}\right) f_{2}$, we have

$$
\begin{aligned}
{\left[\tau_{1}=x_{3} y_{1} f_{1}, \quad \tau_{2}\right.} & =\left(2 \beta f+\left(4 \beta^{3}+8 \beta x_{3}^{2}-y_{1} y_{2}\right) f_{1}\right) /(6 \beta), \\
\tau_{3} & =\left(y_{1} f+2 \beta y_{2} f_{1}\right) /(2 \beta) .
\end{aligned}
$$

From (3.5), $\lambda_{1} \tau_{1}=\mu_{1} \tau_{2}, \lambda_{2} \tau_{1}=\mu_{2} \tau_{3}, \lambda_{3} \tau_{2}=\mu_{3} \tau_{3}$ and so

$$
x_{3} y_{1}=\left(-4 \beta^{3} y_{1}-8 \beta y_{1} x_{3}^{2}+4 \beta^{2} y_{2}+y_{1}^{2} y_{2}\right) /\left(12 \beta^{2}\right)=0 .
$$

If $x_{3}=0$, we get $S_{2}$. The case $y_{1}=0$ leads to $S_{1}$. Q.E.D.

From Lemma 3.1 for $k=1, g^{\prime}$ is regular at $\infty_{i}, i=1,2$, if and only if $2 \beta-x_{1}-$ $2 i y_{3} \neq 0$. Therefore, the subvarieties $R_{i}, i=1,2,3$, correspond to the regular case, and $S_{i}, i=1,2,3$, to the singular one.

Now we can prove the main result of this section.

Theorem 3.1. If $k=1$ and $x_{0}$ has no real periods, then

$$
\left(x_{1}, x_{2}, x_{3}, y_{1}, y_{2}, y_{3}, \beta\right) \in R_{1},
$$

and up to rigid motions, the immersion $x_{0}$ is the minimal once punctured Klein Bottle given in [L2]. 
Proof. Let $u$ denote the meromorphic function given by

$$
u=\frac{2 s^{2}+2 x_{2}-x_{1}^{2}-2 i s y_{1}-2 w}{\left(4 y_{2}^{2}+4 x_{1}^{2} x_{2}-x_{1}^{4}\right)^{1 / 2}}
$$

where $w=v\left(s+\bar{d}_{1}\right)\left(s+\bar{d}_{2}\right)$.

Observe that $u$ has degree two and $[u] \geq \infty_{1} / \infty_{2}^{2}$.

1st case: $\left(x_{1}, x_{2}, x_{3}, y_{1}, y_{2}, y_{3}, \beta\right) \in R_{1}$.

It is clear that $[u]=\infty_{1}^{2} / \infty_{2}^{2}$, and, labelling $r=\left(x_{1}-2 \beta\right) / x_{1} \in \mathbb{R}$, there exists a meromorphic function $z$ satisfying $z^{2} u=(u-r)(r u+1)$. Furthermore,

$$
s(r-1)=\beta z, \quad w(r-1)^{2} u=-\beta^{2} r\left(1+u^{2}\right), \quad 2 \beta d s / w=(1-r) d u /(u z) .
$$

It is straightforward to check that $g^{\prime}=(u+1)(u-r) /((u-1) z)$ and $\eta^{\prime} g^{\prime}=$ $i R\left(u^{2}-1\right) d u / u^{2}, R \in \mathbb{R}$. So, up to rigid motions and change of parameters, $x_{0}$ corresponds to the surface given in [L2]. For completeness, we give a sketch of the proof:

First, note that

$$
\tau_{2}=\frac{8 \beta^{4} r}{3(r-1)^{4}}\left(2 r-3 r^{2}+\left(-1+3 r+r^{2}\right) u\right) \frac{d s}{w}+\frac{4 \beta^{2}\left(-1+3 r-r^{2}\right)}{3(r-1)^{2}} d s
$$

and so,

$$
\tau_{2} \approx \frac{4 \beta^{3} r}{3(r-1)^{4}}\left(2 r-3 r^{2}+\left(-1+3 r+r^{2}\right) u\right) \frac{d u}{u z} .
$$

On the other hand, (3.1) gives $r, \beta,\left(r^{2}-1\right) \neq 0, \infty$, and therefore, from Lemma 3.2, (3.3) and (3.4) hold if and only if:

$$
\int_{\gamma_{1}}\left(2 r-3 r^{2}+\left(-1+3 r+r^{2}\right) u\right) \frac{d u}{u z}=0 .
$$

Defining $t=u r, \zeta=\left(t\left(t-r^{2}\right)(t+1)\right)^{1 / 2}$ and $f: \mathbb{R} \rightarrow \mathbb{R}$ by

$$
f(r)=\int_{-1}^{0}\left(\left(r^{2}+3 r-1\right) t+r^{2}(2-3 r)\right) \frac{d t}{\zeta},
$$

we conclude that $x_{0}$ has no real periods if and only if $f(r)=0$.

If we suppose $\zeta>0$ for $t \in]-1,0[$, it is not hard to see that:

- $f(r)>0$ for $r \in\left[0, r_{1}\left[\right.\right.$, where $r_{1}^{2}+3 r_{1}-1=0$ and $\left.r_{1} \in\right] 0,1 / 2[$.

- $f(1 / 2)<0$.

Therefore, there exist a real root $r_{0}$ of the equation $f(r)=0$ and $\left.r_{0} \in\right] 0,1 / 2[$.

On the other hand, since $d t /\left(\left(t-r^{2}\right) \zeta\right) \approx\left(t-r^{2}\right) d t /\left(\left(r^{4}+r^{2}\right) \zeta\right)$, we have

$$
f^{\prime}(r)=3 \int_{-1}^{0}((r+1) t+r(1-2 r)) d t / \zeta
$$

and so

- $\left.f^{\prime}(r)<0, r \in\right]-\infty, 0[$,

- If $f(r)=0$ and $r>0$, then $f^{\prime}(r)<0$.

It is now easy to conclude that $r_{0}$ is the only real root of $f$ in $\mathbb{R}$. For more details, see [L2].

2nd case: $\left(x_{1}, x_{2}, x_{3}, y_{1}, y_{2}, y_{3}, \beta\right) \in R_{2}$.

As before $[u]=\infty_{1}^{2} / \infty_{2}^{2}$, and, labelling $r^{2}=\left(x_{1}-2 \beta\right) /\left(2 x_{1}\right) \in \mathbb{R}$, there exists a meromorphic function $z$ satisfying $z^{2} u=(u-r)(r u+1)$. Moreover, $s\left(2 r^{2}-1\right)=\beta z$, $w u\left(2 r^{2}-1\right)^{2}=-\beta^{2} r\left(1+u^{2}\right)$ and $2 \beta d s / w=\left(1-2 r^{2}\right) d u /(u z)$. 
On the other hand,

$$
\tau_{2}=a_{1}\left(r+u+2 r^{2} u\right) d s / w+a_{2} d s \approx b_{1}\left(r+u+2 r^{2} u\right) d u /(u z)
$$

where $a_{1}=8 \beta^{4} r\left(6 r^{2}-1\right) /\left(3\left(2 r^{2}-1\right)^{4}\right), a_{2}=4 \beta^{2}\left(6 r^{2}-1\right)\left(1+2 r^{2}\right) /\left(3\left(2 r^{2}-1\right)^{2}\right)$ and $b_{1}=a_{1}\left(1-2 r^{2}\right) /(2 \beta)$.

Analogously,

$$
\tau_{1}=c_{1}\left(r+u+2 r^{2} u\right) d u /(u z)
$$

where $c_{1}=4 \beta^{3} r^{2}\left(1-4 r^{2}\right)^{1 / 2} /\left(1-2 r^{2}\right)^{3}$. Observe that $y_{3} \in \mathbb{R}$ yields $r^{2}\left(1-4 r^{2}\right)>0$. In particular, $r^{2} \in \mathbb{R}$ implies $r \in \mathbb{R}$ and $1-4 r^{2}>0$. Condition (3.1) becomes $\beta, r$, $2 r^{2}-1 \neq 0, \infty$, and so Lemma 3.2, (3.3) and (3.4) imply

$$
\int_{\gamma_{1}}\left(r+u+2 r^{2} u\right) d u /(u z)=0 .
$$

If we define $t=u r$ and $Z=\left(t(t+1)\left(t-r^{2}\right)\right)^{1 / 2}$, the last equation yields $f(r)=0$, where $f: \mathbb{R} \rightarrow \mathbb{R}$ is given by:

$$
f(r)=\int_{-1}^{0}\left(t+r^{2} /\left(1+2 r^{2}\right)\right) d t / Z
$$

In what follows, we will assume that $Z>0$ for $t \in]-1,0[$. Using the fact that $d t /\left(\left(t-r^{2}\right) Z\right) \approx\left(t-r^{2}\right) d t /\left(\left(r^{4}+r^{2}\right) Z\right)$ and integrating by parts, we have

$$
f^{\prime}(r)=\left(2 r /\left(1+2 r^{2}\right)\right) \int_{-1}^{0}\left(t+\left(2 r^{2}+3\right) /\left(2\left(1+2 r^{2}\right)\right)\right) d t / Z
$$

Since $4 r^{2}<1$, then $2 r^{2}+3>2\left(1+2 r^{2}\right)$, and so $r f^{\prime}(r)>0$.

On the other hand, integrating by parts,

$$
f(1 / 2)=2 \int_{-1}^{0}\left(t^{2}+t\right) d t / Z<0
$$

and therefore $4 r^{2}<1$ and $r>0$ imply $f(r)<0$. Since $f(r)=f(-r), f$ has no real roots on $]-1 / 2,1 / 2[$.

3rd case: $\left(x_{1}, x_{2}, x_{3}, y_{1}, y_{2}, y_{3}, \beta\right) \in R_{3}$.

Unfortunately, $[u]=Q \infty_{1} / \infty_{2}^{2}, Q \neq \infty_{1}$. Since $y_{1} \in \mathbb{R}$, then $x_{1}^{2}>8 \beta^{2}$ and so $\left(17 x_{1}-48 \beta\right) / x_{1}>0$. Take $r \in \mathbb{R}$ such that $x_{1} r^{2}=17 x_{1}-48 \beta$. It is clear that (3.1) gives $\left(r^{2}-17\right), \beta, r,\left(25+r^{2}\right)^{2}-36 r^{2} \neq 0, \infty$.

Define $y=\left(1+r^{2}-\left(3\left(15+50 r^{2}-r^{4}\right)\right)^{1 / 2} u\right) /(6 r)$. Thus, there exists a function $z$ on $T$ satisfying $6 r z^{2}=\left(6 r y-25-r^{2}\right)\left(y^{2}-1\right)$. Moreover, a straightforward computation gives

$$
\begin{gathered}
2\left(r^{2}-17\right)\left(6 r y-1-r^{2}\right) s=i y_{1}\left(r^{2}-27\right)\left(23-r^{2}+6 r y\right)-144 i \beta r^{3 / 2} z, \\
\left(r^{2}-17\right)^{2}\left(1+r^{2}-6 r y\right)^{2} w \\
=288 r \beta\left(\beta\left(a_{0}+a_{1} y+a_{2} y^{2}+a_{3} y^{3}\right)+6 r^{1 / 2}\left(r^{2}-17\right) y_{1} z\right),
\end{gathered}
$$

where $a_{0}=-3 r\left(49+r^{2}\right), a_{1}=25+44 r^{2}+r^{4}, a_{2}=-9 r\left(1+r^{2}\right)$ and $a_{3}=18 r^{2}$.

Hence, $24 \beta r^{1 / 2} d s / w=-i\left(r^{2}-17\right) d y / z$.

On the other hand,

$$
\begin{aligned}
\tau_{2}= & 1152 \beta^{4}\left(r^{2}-1\right)\left(-75+3 r^{2}-7 r y-r^{3} y\right) d s /\left(\left(r^{2}-17\right)^{4} w\right) \\
& +4 \beta^{2}\left(r^{2}-1\right)\left(r^{2}+7\right) d s /\left(r^{2}-17\right)^{2}
\end{aligned}
$$


and so

$$
\tau_{2} \approx 48 i \beta^{3}\left(r^{2}-1\right)\left(75-3 r^{2}+7 r y+r^{3} y\right) d y /\left(r^{1 / 2}\left(r^{2}-17\right)^{3} z\right) .
$$

Analogously,

$$
\tau_{3} \approx 24 i \beta^{2} y_{1}\left(75-3 r^{2}+7 r y+r^{3} y\right) d y /\left(r^{1 / 2}\left(r^{2}-17\right)^{2} z\right) .
$$

Hence, (3.3), (3.4) and Lemma 3.2 imply

$$
\int_{\gamma_{1}}\left(75-3 r^{2}+7 r y+r^{3} y\right) d y / z=0
$$

Up to the change of variables $y \rightarrow-y, z \rightarrow i z, r \rightarrow-r$, we will assume that $r<0$. Since $y_{1} \in \mathbb{R},\left(r^{2}+1\right)^{2}>36 r^{2}$ and so $r \in J=\left[-3-8^{1 / 2},-3+8^{1 / 2}\right]$. Thus, the last integral equation is equivalent to

$$
\int_{y_{0}}^{-1}\left(75-3 r^{2}+7 r y+r^{3} y\right) d y / z=0
$$

where $y_{0}=\left(25+r^{2}\right) /(6 r)$. However, if we label $P(y)=75-3 r^{2}+7 r y+r^{3} y$, then $P(-1) P\left(y_{0}\right)=(r-3)\left(6 r-25-r^{2}\right)\left(25+6 r+r^{2}\right)^{2} / 6$ is greater than zero for $r \in J$, and therefore the above integral does not vanish.

4th case: $\left(x_{1}, x_{2}, x_{3}, y_{1}, y_{2}, y_{3}, \beta\right) \in S_{1}$.

If we define $y=u / i$ and label $r=x_{3} / \beta$, then $[y]=\infty_{1}^{2} / \infty_{2}^{2}$ and there exists a meromorphic function $z$ satisfying $z^{2}=(y+r)(r y+1) / y$. Furthermore, $s=\beta z$, $w=\beta^{2} r\left(1-y^{2}\right) / y$ and $2 \beta d s / w=-d y /(y z)$.

In this case,

$$
3 \tau_{2}=8 \beta^{4} r\left(r-y+2 r^{2} y\right) d s / w+4 \beta^{2}\left(2 r^{2}-1\right) d s
$$

and hence

$$
\tau_{2} \approx-4 \beta^{3} r\left(r-y+2 r^{2} y\right) d y /(3 y z)
$$

Since (3.1) becomes $\beta, r, r^{2}-1 \neq 0, \infty$, Lemma 3.2, (3.3) and (3.4) imply

$$
\int_{\gamma_{1}}\left(r-y+2 r^{2} y\right) d y /(y z)=0
$$

that is,

$$
\int_{-r}^{-1 / r}\left(r-y+2 r^{2} y\right) d y /(y z)=0 .
$$

However, $r\left(1-r^{2}\right)\left(r-y+2 r^{2} y\right)>0$ for $y \in[-r,-1 / r]$, and so the last integral never vanishes.

5th case: $\left(x_{1}, x_{2}, x_{3}, y_{1}, y_{2}, y_{3}, \beta\right) \in S_{2}$.

Now, $[u]=Q \infty_{1} / \infty_{2}^{2}, Q \neq \infty_{1}$. Take $r \in \mathbb{R}$ such that $r^{2}=\left(4 \beta^{2}+y_{1}^{2}\right) /\left(4 \beta^{2}+9 y_{1}^{2}\right)$ and observe that condition (3.1) gives $\beta, r, r^{2}-1,\left(1+7 r^{2}\right)^{2}-r^{2}\left(1-25 r^{2}\right)^{2}, 9 r^{2}-1 \neq$ 0 . Define $y=\left(-\left(1+3 r^{2}\right) y_{1}+2 \beta\left(1-34 r^{2}-31 r^{4}\right)^{1 / 2} u\right) /\left(4 r y_{1}\right)$ and observe that there exists a meromorphic function $z$ satisfying:

$$
z^{2}=\left(y^{2}-1\right)\left(y-\left(1+15 r^{2}\right)\left(1-5 r^{2}\right) /\left(4 r\left(r^{2}-1\right)\right)\right) .
$$

A straightforward computation yields

$$
\begin{gathered}
\left(1+3 r^{2}+4 r y\right) s=i y_{1}\left(1-3 r^{2}+(2 r)^{1 / 2} z+2 r y\right), \\
\left(1+3 r^{2}+4 r y\right)^{2}\left(1-9 r^{2}\right) r w=\beta^{2}\left(a z+a_{0}+a_{1} y+a_{2} y^{2}+a_{3} y^{3}\right),
\end{gathered}
$$


where

$$
\begin{gathered}
a=4 r\left(1-r^{2}\right)(2 r)^{1 / 2}\left(1-9 r^{2}\right), \quad a_{0}=2 r\left(-1-18 r^{2}+147 r^{4}\right), \\
a_{1}=-1-21 r^{2}+53 r^{4}+225 r^{6}, \quad a_{2}=6 r\left(r^{2}-1\right)\left(1+3 r^{2}\right), \\
a_{3}=8 r^{2}\left(r^{2}-1\right) .
\end{gathered}
$$

Hence, $y_{1} d s / w=i(2 r)^{1 / 2} d y / z$. Then, it is not hard to check that

$$
\tau_{2}=\beta^{4}\left(r^{2}-1\right)\left(r-25 r^{3}+y+7 r^{2} y\right) d s /\left(6 r^{3}\left(1-9 r^{2}\right) w\right)-\beta^{2}\left(1+7 r^{2}\right) d s /\left(6 r^{2}\right)
$$

and so $12 \tau_{2} \approx i \beta^{2} y_{1}\left(r-25 r^{3}+y+7 r^{2} y\right) d y /\left(2^{1 / 2} r^{5 / 2} z\right)$. Using (3.3), (3.4) and Lemma 3.2, we deduce that

$$
\int_{\gamma_{1}}\left(r-25 r^{3}+y+7 r^{2} y\right) d y / z=0 .
$$

Up to the change of variables $y \rightarrow-y, r \rightarrow-r, z \rightarrow i z$, we can suppose $r<0$. Since $y_{1} \in \mathbb{R}$, then $r^{2} \in[1 / 9,1]$, that is, $r \in J=[-1,-1 / 3]$.

Setting $y_{0}=\left(5 r^{2}-1\right)\left(1+15 r^{2}\right) /\left(4 r\left(1-r^{2}\right)\right)$, it is not hard to prove that $y_{0} \leq 1$ for $r \in J$. Thus, the above integral equation becomes

$$
\int_{y_{0}}^{-1}\left(r-25 r^{3}+y+7 r^{2} y\right) d y / z=0
$$

Define $f: J-\{-1 / 3\} \rightarrow \mathbb{R}$ by

$$
f(r)=\int_{0}^{1}\left(-4 r+4 r^{3}-t+3 r t-7 r^{2} t+21 r^{3} t\right) d t / \zeta
$$

where

$$
\begin{gathered}
t=(y+1) 4 r\left(r^{2}-1\right) /\left((1-3 r)\left(1-r+7 r^{2}+25 r^{3}\right)\right), \\
\zeta=t(1-t)\left(8 r\left(r^{2}-1\right)+(3 r-1)\left(1-r+7 r^{2}+25 r^{3}\right) t\right)^{1 / 2} .
\end{gathered}
$$

Note that $\zeta^{2} \geq 0$ for $t \in[0,1]$ (remember that $r \in J$ ), and hence we can suppose $\zeta \geq 0$ on $[0,1]$. Up to this change of variables, the above integral vanishes if and only if $f(r)=0, r \in J$.

On the other hand, if we define

$$
\begin{gathered}
\tau=\left(-4 r+4 r^{3}-t+3 r t-7 r^{2} t+21 r^{3} t\right) / \zeta \\
l=4\left(1-13 r^{2}+215 r^{4}-75 r^{6}\right) /\left((1+3 r)\left(1-r+7 r^{2}+25 r^{3}\right)\right)
\end{gathered}
$$

and

$$
h=l \zeta /\left(8 r\left(r^{2}-1\right)+(3 r-1)\left(1-r+7 r^{2}+25 r^{3}\right) t\right),
$$

then

$$
\partial \tau / \partial r+d(h)=\left(a_{0}-a_{1} t\right) d t / \zeta
$$

where

$$
\begin{gathered}
a_{0}=4 r\left(-1-7 r-20 r^{2}+76 r^{3}+69 r^{4}+75 r^{5}\right) /\left((1+3 r)\left(1-r+7 r^{2}+25 r^{3}\right)\right), \\
a_{1}=(1+r)\left(1+3 r-98 r^{2}-6 r^{3}+801 r^{4}-1725 r^{5}\right) /\left((1+3 r)\left(1-r+7 r^{2}+25 r^{3}\right)\right) .
\end{gathered}
$$

Therefore,

$$
f^{\prime}(r)=\int_{0}^{1} \partial \tau / \partial r=\int_{0}^{1}(\partial \tau / \partial r+d(h))=\int_{0}^{1}\left(a_{0}-a_{1} t\right) d t / \zeta .
$$


As a consequence, if $r_{0} \in J$ is a root of $f$, then

$$
f^{\prime}\left(r_{0}\right)=R\left(r_{0}\right) \int_{0}^{1} d t / \zeta\left(r_{0}\right)
$$

where

$$
R(r)=\frac{24 r^{3}\left(-17+123 r^{2}-643 r^{4}+25 r^{6}\right)}{\left(9 r^{2}-1\right)\left(1+7 r^{2}\right)\left(-1+r-7 r^{2}-25 r^{3}\right)} .
$$

However, if $r_{1}$ is the only root of $1-r+7 r^{2}+25 r^{3}$ lying in $J$, then it is not hard to see that $R(r)>0$ on ] $-1, r_{1}$ [ and $R(r)<0$ on $] r_{1},-1 / 3$ [. Thus, $f^{\prime}\left(r_{0}\right)>0$ if $\left.r_{0} \in\right]-1, r_{1}\left[\right.$ and $f^{\prime}\left(r_{0}\right)<0$ if $\left.r_{0} \in\right] r_{1},-1 / 3[$.

Straightforward computations yield $f(-1)<0, \operatorname{Limit}_{r \rightarrow-1 / 3} f=-\infty$ and $f\left(r_{1}\right)$ $=-\left(1+5 r_{1}+7 r_{1}^{2}-29 r_{1}^{3}\right) \pi /\left(32 r_{1}\left(r_{1}^{2}-1\right)\right)^{1 / 2}<0$. Now it is easy to conclude that $f$ has no real roots on $J$.

6th case: $\left(x_{1}, x_{2}, x_{3}, y_{1}, y_{2}, y_{3}, \beta\right) \in S_{3}$.

Observe that $[u]=\infty_{1}^{2} / \infty_{2}^{2}$. Since $x_{2} \in \mathbb{R}$, then $4 x_{3}^{2}>y_{2}^{2}$. If we take $j \in \mathbb{R}$ such that $y_{2} j+2 x_{3}^{2}=\left(4 x_{3}^{4}-y_{2}^{2}\right)^{1 / 2}$, there exists a meromorphic function $z$ satisfying $2 j u z^{2}=2 j\left(u^{2}-1\right)+\left(1-3 j^{2}\right) u$. Moreover, it is not hard to check that:

$$
\begin{gathered}
\left(1+j^{2}\right)^{1 / 2} s=(2 j)^{1 / 2} x_{3} z, \quad\left(1+j^{2}\right) u w=-2 j x_{3}^{2}\left(1+u^{2}\right), \\
(8 j)^{1 / 2} x_{3} d s / w=-\left(1+j^{2}\right)^{1 / 2} d u /(u z) .
\end{gathered}
$$

A straightforward computation gives

$$
3 \tau_{2}=\frac{32 x_{3}^{4} j}{\left(1+j^{2}\right)^{2}} \frac{(u-j) d s}{w}+\frac{8 x_{3}^{2}}{1+j^{2}} d s \approx \frac{8 x_{3}^{3}(2 j)^{1 / 2}}{\left(1+j^{2}\right)^{3 / 2}} \frac{(j-u) d u}{u z} .
$$

Condition (3.1) gives $j, x_{3} \neq 0, \infty$, and thus Lemma 3.2, (3.3) and (3.4) yield

$$
\int_{\gamma_{1}}(u-j) d u /(u z)=0
$$

that is,

$$
\int_{u_{0}}^{0}(u-j) d u /(u z)=0
$$

where $u_{0}=\left(-1+3 j^{2}-\left(1+10 j^{2}+9 j^{4}\right)^{1 / 2}\right) /(4 j)$.

However, for $u \in\left[u_{0}, 0\right]$ we have $j(u-j)<0$, and then the above integral does not vanish. Q.E.D.

Return to the general case $k>1$. For the remainder of this section, we will assume that $k$ is even.

Consider the symmetry group $\operatorname{Sym}(N)$ of $x: N \rightarrow \mathbb{R}^{3}$. Each $\bar{J} \in \operatorname{Sym}(N)$ extends conformally to a diffeomorphism on $\bar{N}$ fixing $\bar{P}_{1}$, which we will call $\bar{J}$ too. Then, there exists a unique $J \in \operatorname{Aut}(\bar{M})$ satisfying $\pi_{0} \cdot J=\bar{J} \cdot \pi_{0}$ and $J \cdot I=I \cdot J$. It is clear that $J\left(\left\{P_{1}, I\left(P_{1}\right)\right\}\right)=\left\{P_{1}, I\left(P_{1}\right)\right\}$. Define $G=\{J: \bar{J} \in \operatorname{Sym}(N)\}$ and observe that $G$ is a subgroup of $\operatorname{Aut}(\bar{M})$ isomorphic to $\operatorname{Sym}(N)$.

Theorem 3.2. If $\operatorname{Sym}(N)$ is not trivial, then it contains either two or four elements. Furthermore, if \# $\operatorname{Sym}(N)=4$, then $A\left(P_{1}\right)=I\left(P_{1}\right)$. 
Proof. Define $q=\operatorname{Order}(\operatorname{Sym}(N))$. Remember that Proposition 2.2 implies $g=$ $h(s-\bar{\alpha}) /(s+\alpha)$. Moreover, if we set $\Delta=\{X \in \bar{M}: A(X) \neq X, g(X)=g(A(X))\}$, then from the demonstration of Proposition 2.2 it is clear that $\Delta=s^{-1}(\{\alpha,-\alpha\})$ and contains four different points $\left\{Q_{0}, I\left(Q_{0}\right), A\left(Q_{0}\right), I\left(A\left(Q_{0}\right)\right\}\right.$. On the other hand, for each $J \in \operatorname{Aut}(\bar{M}), J \cdot A=A \cdot J$ (see [FK, p. 108]), and so $s \cdot J=L \cdot s$, where $L$ is a Möbius transformation. Moreover, if $J \in G$ and $R$ is the rigid motion that determines $J$, we have $g \cdot J=R \cdot g$. Therefore, if $X \in \Delta, g(J(X))=$ $R(g(X))=R(g(A(X)))=g(J(A(X)))=g(A(J(X)))$, which implies $J(X) \in \Delta$. Hence $J(\Delta)=\Delta$, for each $J \in G$.

Furthermore, if $u=(s-\alpha) /(s+\alpha)$ and $J \in G$, then either $u \cdot J=d / u$ or $u \cdot J=c u, d, c \in \mathbb{C},|c|=1$. In particular, since $g \cdot J=R \cdot g$ (where $R$ is a rigid motion) we deduce that either $g \cdot J=\theta / g$ or $g \cdot J=\theta g,|\theta|=1$. Label $H=\{J \in G: g \cdot J=\theta g,|\theta|=1\}=\{J \in G: u \cdot J=c u,|c|=1\}$ and observe that $H$ is a cyclic subgroup of $G$ whose order $p$ is either $q$ or $q / 2$. It is clear that $J\left([h]_{0}\right)=[h]_{0}$, for each $J \in H$. Take $J_{0}$ a generator of $H$.

Since $J_{0} \cdot A=A \cdot J_{0}$ and $\bar{J}_{0}$ fixes $\bar{P}_{1}$, we have $J_{0}(D)=D, J_{0}(A(D))=A(D)$, where $D=P_{1} I\left(P_{1}\right)$.

We will distinguish three cases:

(a) $A\left(P_{1}\right) \neq P_{1}, I\left(P_{1}\right)$. Suppose $p \geq 2$.

Since $J_{0} \in H$, then $u \cdot J_{0}=c u,|c|=1$. If $J_{0}\left(P_{1}\right)=I\left(P_{1}\right)$, then $u\left(J_{0}\left(P_{1}\right)\right)=$ $u\left(I\left(P_{1}\right)\right)$, that is, $c u\left(P_{1}\right)=1 / \bar{u}\left(P_{1}\right)$, which implies $\left|u\left(P_{1}\right)\right|^{2}=1 / c=1$. In particular, $A\left(I\left(P_{1}\right)\right)=P_{1}$, a contradiction. Hence, $J_{0}\left(P_{1}\right)=P_{1}$ and $J_{0}\left(I\left(P_{1}\right)\right)=I\left(P_{1}\right)$. Therefore we obtain $c u\left(P_{1}\right)=u\left(P_{1}\right), c / \bar{u}\left(I\left(P_{1}\right)\right)=1 / \bar{u}\left(P_{1}\right)$. Since $c=1$ and $p \geq 2$ imply $J_{0}=A$, which is absurd from our hypothesis in (a), we deduce that $u\left(P_{1}\right) \in\{0, \infty\}$ and thus $P_{1} \in \Delta$. From (3.2) and Lemma 2.1 for $P=A\left(P_{1}\right), p$ is odd and we have $[h]_{0} \geq P_{1}^{2}$. Since $\nu_{1}=3$, then $[\eta g]_{\infty} \leq P_{1} I\left(P_{1}\right)$ (see (1.4)). From Remark 2.1, Residue $\left[\eta g, P_{1}\right]=0$, and therefore $\eta g$ is holomorphic. Since $\eta g$ has no real periods, the elementary theory of compact Riemann surfaces (see [FK]) gives $\eta g=0$, a contradiction.

(b) $A\left(P_{1}\right)=P_{1}$. Assume that $p \geq 2$.

Suppose $J_{0}\left(P_{1}\right)=P_{1}$. Define $z=\left(s-s\left(P_{1}\right)\right) /\left(s+\bar{s}\left(P_{1}\right)\right)$. Then, taking into account Lemma 2.2 and using its notation for $P=P_{1}$, up to rigid motions and relabelling of the Weierstrass point set of $\bar{M}, h=\mu x,|\mu|=1$. Consider first the case $J_{0} \neq A$, that is, $p>2$. Hence, $z \cdot J_{0}=\theta z, \theta \neq 1$. As $u \cdot J_{0}=c u$, $|c|=1$ and $u=L \cdot z$, where $L$ is a Möbius transformation, we obtain $u=b z$, $|b|=1$, and thus $g=\lambda z x,|\lambda|=1$. Therefore, as in the case (a), $\eta g$ is holomorphic once again, a contradiction. Suppose now $J_{0}=A$, that is, with the notation of Lemma $2.2, \theta=-1, z \cdot J_{0}=z$ and $p=2$. In this case, $u=b(z-\beta) /(\bar{\beta} z-1)$, where $|b|=1$ and $|\beta| \neq 1$. Using that $\nu_{1}=3$ (see (1.4)), it is not hard to check that $\eta g=i(z-\beta)(\bar{\beta} z-1) d z / z^{2}$, and so Residue $\left[\eta g, P_{1}\right] \neq 0$, which is absurd.

Hence, $J_{0}\left(P_{1}\right)=I\left(P_{1}\right)$. The ideas of Lemma 2.2 and the fact that $J_{0} \cdot I=I \cdot J_{0}$ yield, up to changes of variables, $z \cdot J_{0}=1 / z$. Moreover, $J_{0} \in H$ gives

$$
J_{0}\left(Q_{0} A\left(Q_{0}\right)\right)=Q_{0} A\left(Q_{0}\right) .
$$

Thus $z\left(Q_{0}\right)=z\left(J_{0}\left(Q_{0}\right)\right)=1 / z\left(Q_{0}\right)$, and so $z\left(Q_{0}\right)^{2}=1$. In particular, $Q_{0} A\left(Q_{0}\right)=$ $I\left(Q_{0}\right) A\left(I\left(Q_{0}\right)\right)$ and $u$ is constant, which is absurd.

(c) $A\left(P_{1}\right)=I\left(P_{1}\right)$. In this case, Lemma 2.3 implies that $G$ is a subgroup of $\{\operatorname{Id}, A, B, A \cdot B\}$. Furthermore, if $q=4$ it is easy to prove that $H=\{\operatorname{Id}, A\}$. Q.E.D. 
Corollary 3.1. If $k=2$, then $\operatorname{Sym}(N)$ contains at most two elements.

Proof. If $q=4$, from the proof of Theorem $3.2 A\left(P_{1}\right)=I\left(P_{1}\right)$. Using the notation of Lemma 2.3 we have $G=\{\mathrm{Id}, A, B, A \cdot B\}$. Up to change of variables, $B(s, v)=$ $(-s,-1 / v)$ and $P_{1}=\infty_{1}$. Therefore, $a=d_{1}+d_{2}+d_{3}, b=d_{1} d_{2}+d_{1} d_{3}+d_{2} d_{3}$ and $c=d_{1} d_{2} d_{3}$ are real numbers. If we define $P(s)=s^{3}-a s^{2}+b s-c$ and $Q(s)=-P(-s)$, then $\bar{M}=\left\{(s, v) \in(\mathbb{C} \cup\{\infty\})^{2}: v^{2}=P(s) / Q(s)\right\}$. Furthermore, up to rigid motions, change of parameters and relabellings, from the definition of $G$ and Propositions 2.2, 2.3 we get

$$
g=(s-\alpha) v /(s+\alpha), \quad \eta g=i\left(s^{2}-\alpha^{2}\right) d s,
$$

where $\alpha \in \mathbb{R}-\{0\}$.

It is clear that $2 \phi_{2}=\left((s-\alpha)^{2} v^{2}+(s+\alpha)^{2}\right) d s / v$. If $P(s)$ has three real roots $d_{1}, d_{2}, d_{3}$ and, for instance, $\left|d_{1}\right|<\left|d_{2}\right|<\left|d_{3}\right|$, from Remark 2.1 we have

$$
\int_{-\left|d_{3}\right|}^{-\left|d_{2}\right|} \phi_{2}=0
$$

which is absurd (observe that $(s-\alpha)^{2} v^{2}+(s+\alpha)^{2}>0$ on $\left.\left[-\left|d_{3}\right|,-\left|d_{2}\right|\right]\right)$.

Hence, both $P(s)$ and $Q(s)$ have only one real root, for instance, $d_{1}$ and $-d_{1}$ respectively. Up to the change $s \rightarrow s / d_{1}$, we will suppose that $d_{1}=1$.

On the other hand, Remark 2.1 once again gives Residue $\left(\phi_{1}, \infty_{1}\right)=0$, that is:

$$
a^{3}-2 a b+2 c+2 \alpha a^{2}+2 \alpha^{2} a=0 .
$$

Since $P(1)=0$, we have $b=a+c-1$, and so the last equation gives

$$
2(a-1) c=a\left((a+\alpha-1)^{2}+(1+\alpha)^{2}\right) .
$$

Therefore,

$$
2(a-1)(a+c)=a\left((a+\alpha)^{2}+\alpha^{2}\right) .
$$

On the other hand, as $P(s)=(s-1)\left(s^{2}+(1-a) s+c\right)$ has only one real root, $s^{2}+(1-a) s+c$ has no real roots, and hence $(a-1)^{2}<4 c$. So, $2 c=$ $a\left((a+\alpha-1)^{2}+(1+\alpha)^{2}\right) /(a-1)>0$, and thus $a /(a-1)>0$. Therefore, $2(a+c)=a\left((a-\alpha)^{2}+\alpha^{2}\right) /(a-1)>0$. As a consequence, $a s^{2}+c>0$, for each $s \in[-1,1]$.

To finish the proof, note that

$$
2 \phi_{1}=i R(s) d s / v
$$

where $R(s)=(s+\alpha)^{2}-(s-\alpha)^{2} v^{2}$.

A easy computation gives $R(s)=(s-\alpha)^{2}\left(a s^{2}+c\right) / Q(s)+4 \alpha s$, and taking into account that $Q(s)>0$ for each $s \in]-1,1[$, we deduce that

- $R(s)>0$ if $s \in[0,1[$ and $\alpha>0$,

- $R(s)>0$ if $s \in]-1,0]$ and $\alpha<0$.

Remark 2.1 yields

$$
\int_{-1}^{1} R(s) d s / v=0
$$


However, $\int_{-1}^{1} R(s) d s / v=2 \int_{0}^{1} R(s) d s / v=2 \int_{-1}^{0} R(s) d s / v$, and this integral obviously never vanishes. Q.E.D.

We say that a complete nonorientable minimal surface $N$ in $\mathbb{R}^{3}$ has trivial topology if $N=\mathbb{R P}^{2}-\left\{\bar{P}_{1}, \ldots, P_{r}\right\}$. A complete study of these surfaces with low total curvature can be found in [I1], [K1], [K2] and [S].

As a consequence of our results, we have

Corollary 3.2. The only complete nonorientable minimal surface with total curvature greater than $-12 \pi$, nontrivial topology and at least three symmetries is the once punctured Klein Bottle described in Theorem 3.1.

The proof is an easy consequence of the congruence formula of Meeks, the JorgeMeeks formula, the nonexistence theorem of Kusner in [K1], Theorem 3.1 and Corollary 3.1 .

\section{REFERENCES}

[B] E. L. Barbanel, Complete minimal surfaces in $\mathbb{R}^{3}$ of low total curvature, Thesis, Univ. of Massachusetts, 1987.

[BA] A. Alves de Barros, Complete nonorientable minimal surfaces in $\mathbb{R}^{3}$ with finite total curvature, An. Acad. Bras. Cienc. 59 (1987), 141-143. MR 89h:53017

[BL] D. Bloss, Elliptische Funktionen und vollständige Minimalflächen, Thesis, Freie Universität Berlin, Berlin, 1989. Cf. MR 94j:53003

[CG] C. C. Chen and F. Gackstatter, Elliptische und Hyperelliptische Functionen und vollständige minimalflächen von Enneperschen Typ, Math. Ann. 259 (1982), 359-369. MR 84d:53005

[FK] H. M. Farkas and I. Kra, Riemann surfaces. Graduate Texts in Math., 71, Springer-Verlag, Berlin, 1980. MR 82c:30067

[HM] D. Hoffman and W. H. Meeks III, Embedded minimal surfaces of finite topology, Ann. of Math. 131 (1990), 1-34. MR 91i:53010

[I1] T. Ishihara, Complete Möbius strips minimally immersed in $\mathbb{R}^{3}$, Proc. Amer. Math. Soc. 107 (1989), 803-806. MR 90c:53024

[I2] _ Complete nonorientable minimal surfaces in $\mathbb{R}^{3}$, Trans. Amer. Math. Soc. 333 (1992), 889-901. MR 92m:53013

[JM] L. Jorge and W. H. Meeks III, The topology of complete minimal surfaces of finite total Gaussian curvature, Topology 22 (1983), 203-221. MR 84d:53006

[K1] R. Kusner, Conformal geometry and complete minimal surfaces, Bull. Amer. Math. Soc. (N.S.) 17 (1987), 291-295. MR 88j:53008

[K2] _ Global geometry of extremal surfaces, Dissertation, Univ. of California, Berkeley, 1988.

[L1] F. J. Lopez, The classification of complete minimal surfaces with total curvature greater than $-12 \pi$, Trans. Amer. Math. Soc. 334 (1992), 49-74. MR 93a:53008

[L2] _ A complete minimal Klein Bottle in $\mathbb{R}^{3}$, Duke Math. J. 71 (1993), 23-30. MR 94e:53005

[LM] F. J. Lopez and F. Martin, Complete nonorientable minimal surfaces and symmetries, Duke Math. J. 79 (1995), 667-686.

$[\mathrm{M}]$ W. H. Meeks, The classification of complete minimal surfaces in $\mathbb{R}^{3}$ with total curvature greater than $-8 \pi$, Duke Math. J. 48 (1981), 523-535. MR 82k:53009

[OL] M. E. G. G. de Oliveira, Some new examples of nonorientable minimal surfaces, Proc. Amer. Math. Soc. 94 (1986), 629-636. MR 87m:53008

[OT] M. E. G. G. de Oliveira and E. Toubiana, Surfaces nonorientables de genre deux, Bol. Soc. Brasil. Mat. (N. S.) 24 (1993), 63-88. MR 94f:53011

[OS] R. Osserman, A survey of minimal surfaces, 2nd ed., Dover, New York, 1986. MR 87j:53012

[R] M. Ross, Complete nonorientable minimal surfaces in $\mathbb{R}^{3}$, Comment. Math. Helv. 67 (1992), 64-76. MR 92k:53022

[S] N. Schmitt, Minimal surfaces with flat ends, Dissertation, Univ. of Massachusetts at Amherst, 1992-1993. 
[SM] A. J. Small, Minimal surfaces in $\mathbb{R}^{3}$ and algebraic curves, Differential Geom. Appl. 2 (1992), 309-384. MR 94h:53014

[T] E. Toubiana, Surfaces minimales non orientables de genre quelconque, Bull. Soc. Math. France 121 (1993), 183-197. MR 94c:53013

Departamento de Geometría y Topología, Facultad de Ciencias, Universidad de Granada, 18071-Granada, Spain

E-mail address: fjlopez@goliat.ugr.es 